\title{
Temperature Correction of Radiometric and Geometric Models for an Uncooled CCD Camera in the Near Infrared
}

\author{
Thierry Sentenac, Yannick Le Maoult, Guy Rolland, and Michel Devy
}

\begin{abstract}
This paper presents radiometric and geometric models for both temperature and displacement noncontact measurements using an uncooled charge-coupled device (CCD) video camera. Such techniques ("one sensor-two measures") represent an interest in many industrial low cost applications and scientific domains.

To benefit from both measurements, we have to use the camera's spectral response in the near infrared spectral band from 0.75 to $1.1 \mu \mathrm{m}$. In this spectral band, the temperature variations of an uncooled CCD camera are taken into account in the radiometric and geometric models. By using physical models for CCD camera, we quantify detector's quantum efficiency, sensor noise and spatial resolution as a function of the wavelength and of the detector temperature. These models are confirmed by experimental results of calibration with a low cost uncooled camera based on a Sony detector and operating over the detector temperature range of $-\mathbf{3 0}$ to $-\mathbf{5 0}$.
\end{abstract}

Index Terms-Camera calibration, charge-coupled device (CCD) cameras, geometric model, modulation transfer function (MTF), noise estimation, radiometric model, sensor modeling, spatial resolution, temperature.

\section{INTRODUCTION}

$\mathbf{I}$ $\mathrm{N}$ RECENT YEARS, the material research center of the Ecole des Mines d'Albi-Carmaux (CROMeP) has developed temperature gradient measurements based on infrared thermographic cameras [1]. At the same time, a team has been involved in three-dimensional (3-D) deformation [2] and distortion [3] measurements using charge-coupled device (CCD) cameras. To improve maintenance and cost in industrial applications, we have to measure both temperature and dimensional characteristics using only one noncontact sensor. The system must be able to measure a temperature from 350 to $1000{ }^{\circ} \mathrm{C}$ with a tolerance of $\pm 25^{\circ} \mathrm{C}$ and spatial surface deformations of $\pm 0.1 \mathrm{~mm}$ with an observation distance of $1 \mathrm{~m}$.

However, the main difficulty is the temperature measurement. Adopted from the spectral photon luminance $L_{s}$ of a real source

T. Sentenac and Y. Le Maoult are with the Ecole des Mines d'Albi-Carmaux, Albi, France (e-mail: sentenac@enstimac.fr, le.maoult@enstimac.fr).

G. Rolland is with the Centre National d'Etudes Spatiales, Toulouse, France (e-mail: guy.rolland@cnes.fr).

M. Devy is with the Laboratoire d'Analyze et d'Architecture des Systèmes (LAAS), Toulouse, France (e-mail: michel@laas.fr).
[4], the sensor operates in the infrared spectral band. The luminance is described by the Planck's radiation law, as follows:

$$
L_{s}(\lambda, T)=\varepsilon \frac{C_{3} \lambda^{4}}{\exp \left(\frac{C_{2}}{\lambda T}\right)-1} \quad\left(\text { photons } / \mathrm{s}-m^{2}-\mathrm{sr}-\mu \mathrm{m}\right)
$$

where $T$ is the absolute temperature (Kelvin), $\varepsilon$ is the emissivity of the source, $h$ is Planck's constant $\left(h=6.6210^{-34} \mathrm{Js}\right), c$ is the speed of light ( $c=310^{8} \mathrm{~ms}$ ) and $k$ is Botzmann's constant $\left(k=1.380710^{-23} \mathrm{~J} / K\right)$. Finally, the second and the third radiation constants are respectively given by $C_{2}=h c / k \mu m K$ and $C_{3}=2 c$.

Thus, applying relation (1) when temperature $T$ equals $350{ }^{\circ} \mathrm{C}$ (and, respectively, $1000{ }^{\circ} \mathrm{C}$ ), the luminance reaches a peak at a wavelength value of $4.65 \mu \mathrm{m}$ (and respectively $2.25 \mu \mathrm{m}$ ). But, the traditional 3-5 $\mu \mathrm{m}$ infrared cameras, are often limited by their fragility, high cost and low spatial resolution. In the near infrared spectral band (NIR) $(0.75-1.1 \mu \mathrm{m})$, luminance values (from $10^{16}$ to $2 \times 10^{21}$ photons $/ \mathrm{sm}^{2} \mathrm{sr} \mu \mathrm{m}$ ) allow a measurement of a temperature from 350 to $1000{ }^{\circ} \mathrm{C}$. Moreover, CCD cameras operating in NIR spectral band are relatively low cost, light weight, compact and have high spatial resolution. They can measure through a glass window and present less sensitivity to uncertainties concerning the emissivity of the object. A few industrial applications with low cost CCD cameras related to high-temperature measurement over the range $\left[800-1800^{\circ}\right.$ ] are reported in [5] and [6]. In this paper, our objective is to measure temperatures below the usual value of $800^{\circ}$, in low illuminated conditions with noise considerations like in [7]. Additionally, the new radiometric model presented in this paper takes into account the variation of the detector temperature of low cost uncooled CCD devices widely used in industrial situations. This radiometric model is based on the study of CCD sensor properties like quantum efficiency and noise. Moreover, we examine the spatial resolution changes in NIR spectral band for the same range of temperatures. From the study of the modulation transfer function (MTF), we deduce the influence of wavelength and detector temperature on the intrinsic parameters required in the "pin-hole" geometric model [8] used in our applications. Finally, after characterization and calibration procedures, some advantages and limitations of this strategy of "one sensor-two measurements" are discussed.

\section{RADIOMETRIC MODEL}

The radiometric model is determined by a formal relationship between the temperature $T$ of an ideal blackbody source and the 


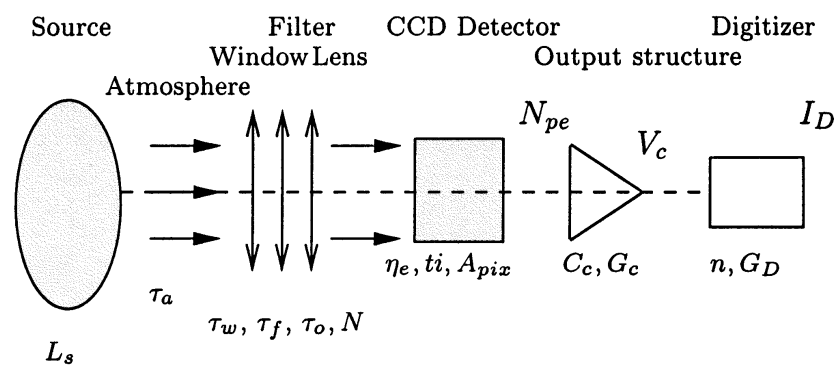

Fig. 1. Temperature calibration situation.

analog $V_{c}$ or digital $I_{D}$ camera CCD output. Usually, the model is used with infrared cameras and can be directly applied with cooled CCD cameras. However, uncooled cameras are characterized by a temperature detector variation $T_{d}$ which involves sensor noise and quantum efficiency $\eta_{e}$ modifications mainly due to the variation of the absorption light. As a result, the model parameters depend on detector temperature and a temperature calibration allows to determine their values. In this section, we present results in the case of a camera based on a Sony detector.

\section{A. Temperature-Signal Relation}

Fig. 1 illustrates an imaging COD directly viewing a hot source with a photon luminance $L_{s}$ during integration time $t i$. Ideally, such a system produces a number of photoelectrons $N_{p e}$ at a collection site given by

$$
N_{p e}=K_{o} t i \int_{\Delta \lambda} \cdot \tau_{a}(\lambda) \tau_{w}(\lambda) \tau_{f}(\lambda) \tau_{o}(\lambda) L_{s}(\lambda) \eta_{e}\left(\lambda, T_{d}\right) d \lambda
$$

where $K_{o}$ is the optical focused magnification $\left(K_{o}=\right.$ $\left.\left(\pi A_{p i x}\right) /\left(4 N^{2}\right)\right), N$ is the F-number and $A_{p i x}$ is the sensitive pixel area. $\tau_{a}, \tau_{w}, \tau_{f}$, and $\tau_{o}$ are respectively atmospheric, window glass, near infrared filter and optical system transmittance. $\eta_{e}$ is the detector's quantum efficiency $\left(e^{-}\right)$/photons. Such a model can be improved: for example [9] describes nonlinearities in short exposure time $(<40 \mathrm{~ms})$ due to the mechanical nature of the shutter mechanism.

The charge packets integrated at each collection site are transfered in parallel with a charge transfer efficiency $(\mathrm{CTE} \approx 1)$ into an output register. Before the next parallel transfer, the register transfers each of its charge packets to a floating diode acting as a capacitor $C_{c}$. The difference voltage $V_{c}$ of the capacitor is amplified with a gain $G_{c}$ and the final value is linearly proportional to the number of electrons $N_{p e}$

$$
V_{c}=\frac{G_{c}}{C_{c}} q N_{p e}\left(\lambda, T_{d}\right)
$$

where $q$ is the electronic charge ( $q=1.610^{-19}$ Coulomb).

Finally, the signal generated by the camera CCD (full range value is named $V_{c F R}$ ) is converted into a digital signal $I_{D}$ with a resolution of $n$ bits and is amplified with a gain $G_{D}$

$$
I_{D}=\frac{G_{D} 2^{n}}{V_{c F R}} V_{c}\left(\lambda, T_{d}\right) .
$$

Relation (4) shows that the output signal $I_{D}$ depends on the spectral quantum efficiency and on transmission and source

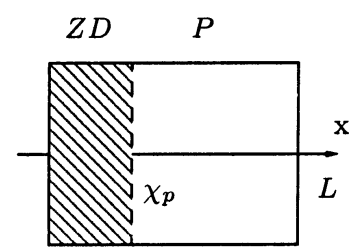

Fig. 2. Cross section of photomos structure.

characteristics. From this relation, the radiometric model can be determined using the following steps.

1) In the case of a CCD detector operating in NIR spectral band, a very small wavelength range $\Delta \lambda$ equals to $0.35 \mu \mathrm{m}$ is selected. The integral can then be computed around an effective wavelength $\lambda_{e} . L_{s}$ will only vary in the source temperature $T\left(L_{s}\left(\lambda_{e}, T\right)\right)$ and $\eta_{e}$ depends only on the detector temperature $\left[\left(\eta_{e}\left(\lambda_{e}, T_{d}\right)\right]\right.$.

2) During the temperature calibration procedure with a controlled atmosphere and with the same window, filter, and optical system, we can consider transmittances $\tau_{a}, \tau_{w}, \tau_{f}$, and $\tau_{o}$ as constants. In our system, only F-number $N$, exposure time, $t i$ and analog-to-digital converter gain $G_{D}$ can be modified

$$
I_{D}=A_{1} \frac{G_{D} t i}{N^{2}} L_{s}\left(\lambda_{e}, T\right) \eta_{e}\left(\lambda_{e}, T_{d}\right)
$$

where $\quad A_{1}$ is a constant $\left(A_{1}=\tau_{a} \tau_{w} \tau_{f} \tau_{o}\left(2^{n} / V_{c F R}\right)\left(G_{c} / C_{c}\right) q\left(\pi A_{p i x} / 4\right)\right)$.

3) The luminance $L_{s}\left(\lambda_{e}, T\right)$ is described by the Planck's blackbody radiation law (1) with an emissivity $\varepsilon_{B B} \approx 1$

$$
I_{D}=\frac{A_{2} \frac{G_{D} t i}{N^{2}}}{\exp \left(\frac{B}{T}\right)-1} \eta_{e}\left(\lambda_{e}, T_{d}\right)
$$

where $A_{2}$ and $B$ are constants $\left(A_{2}=A_{1} \varepsilon_{B B} C_{3} \lambda_{e}^{-4}\right.$ and $B=C_{2} / \lambda_{e}$.

4) Moreover, we introduce in relation (6) a variable $D$ to model continuous value of camera output in dark environment. The value of $D$ also depends on the effect of noise sources of the CCD imaging system which are highly linked to the detector temperature.

Finally, the temperature-signal relation is described by

$$
I_{D}=\frac{A_{2} \frac{G_{D} t i}{N^{2}} \eta_{e}\left(\lambda_{e}, T_{d}\right)}{\exp \left(\frac{B}{T}\right)-1}+D\left(T_{d}\right)
$$

\section{B. Detector's Quantum Efficiency Model $\eta_{e}$}

The detector's quantum efficiency is defined as the ratio of electron flux $N_{p e}$ to incident photon flux $N_{p}$ onto the optical system for a single collection site and a particular exposure time

$$
\eta_{e}\left(\lambda_{e}, T_{d}\right)=\frac{N_{p e}\left(\lambda_{e}, T_{d}\right)}{N_{p}} .
$$

In photomos devices, we can approximate the quantum efficiency by that of an abrupt half junction. We assume that the front part of photomos structure is close to zero (see Fig. 2). 


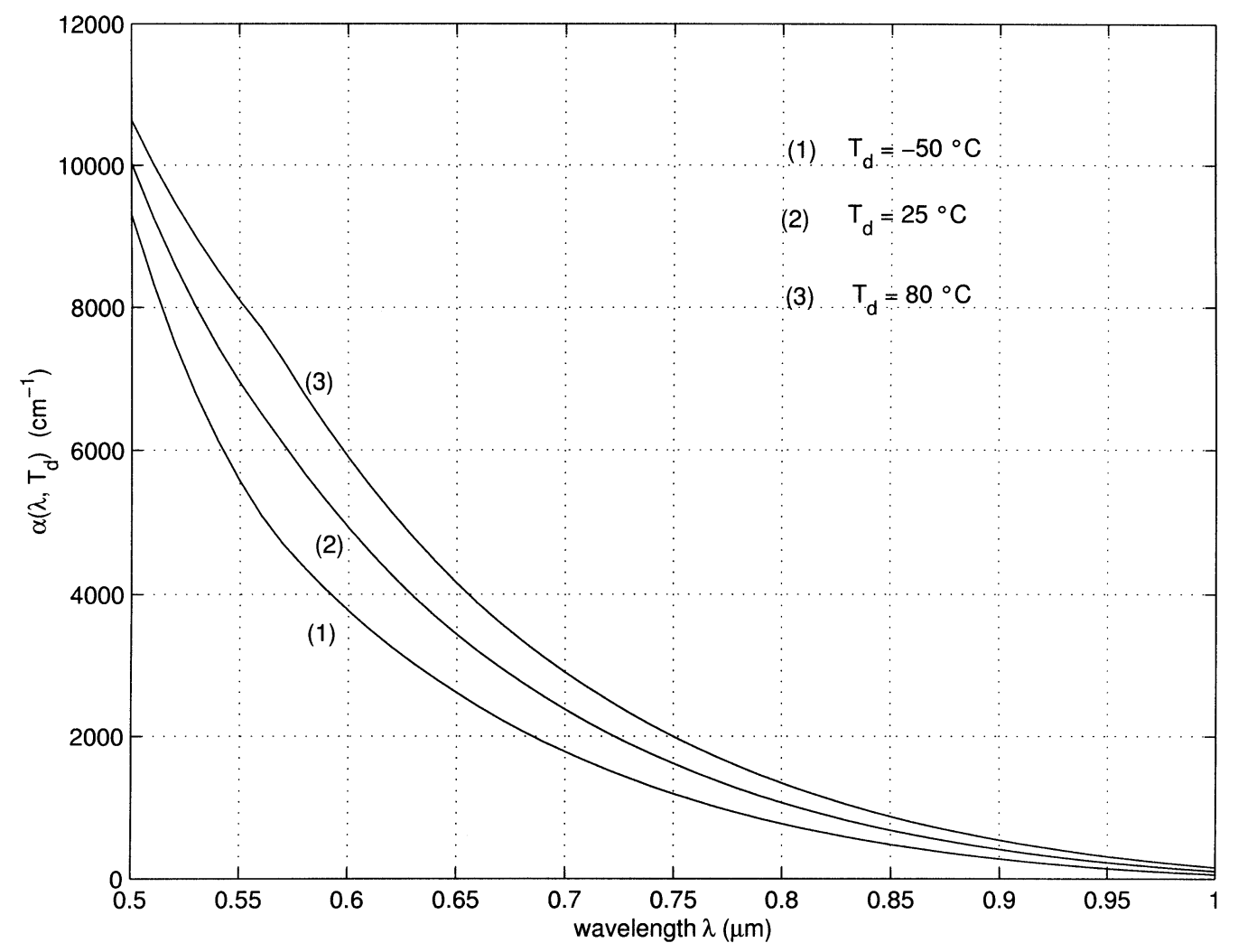

Fig. 3. $\alpha$ as a function of $\lambda$ and $T_{d}$.

The quantum efficiency is then defined by the sum of the depletion region $Z D$ efficiency $\left(\eta_{Z D}\right)$ and back region $P$ efficiency $\left(\eta_{P}\right)$. With reference [10], $\eta_{Z D}$ and $\eta_{P}$ are given by

$$
\begin{aligned}
\eta_{Z D}= & 1-\exp \left(-\alpha \mathcal{X}_{p}\right) \\
\eta_{P}= & \frac{\left(\alpha L_{n}\right)^{2}}{\left(\alpha L_{n}\right)^{2}-1}\left(\exp \left(-\alpha \mathcal{X}_{p}\right)\right. \\
& \left.+\frac{\exp (-\alpha L)-\exp \left(-\alpha \mathcal{X}_{p}\right) \cosh \left(\frac{L-\mathcal{X}_{p}}{L_{n}}\right)}{\alpha L_{n} \sinh \left(\frac{L-\mathcal{X}_{p}}{L_{n}}\right)}\right)
\end{aligned}
$$

where $L_{n}, L$, and $\chi_{p}$ are respectively electron diffusion length in region $P(\mathrm{~mm})$, substrate length $(\mathrm{mm})$, and limit of depletion region $(\mathrm{mm}) . \alpha$ represents the absorption photons coefficient in silicon part (see formula in Appendix). Fig. 3 shows the evolution of this coefficient versus the wavelength at different temperatures. It decreases with an increasing wavelength and a decreasing temperature.

Therefore, the quantum efficiency of the detector is defined as a function of the wavelength and the temperature owing to absorption coefficient characteristics

$$
\begin{aligned}
\eta_{e}= & +\exp (-\alpha L)\left(\frac{K_{a}}{K_{b}}\right) \\
& +\exp \left(-\alpha \mathcal{X}_{p}\right)\left(\frac{K_{a}}{K_{b}}\left(K_{b}-\frac{K_{b}}{K_{a}}-\cosh \left(\frac{L-\mathcal{X}_{p}}{L_{n}}\right)\right)\right)
\end{aligned}
$$

where $K_{a}$ and $K_{b}$ are variable $\left(K_{a}=\left(\alpha L_{n}\right)^{2} /\left(\alpha L_{n}\right)^{2}-1\right.$ and $\left.K_{b}=\alpha L_{n} \sinh \left(\left(L-\chi_{p}\right) /\left(L_{n}\right)\right)\right)$.
To model the quantum efficiency of the detector, we have to determine the internal parameters $\left(L_{n}, L\right.$, and $\left.\chi_{p}\right)$ of the detector. However, these parameters can generally not be accessed easily. One solution can be to measure the quantum efficiency and to fit the parameters of model (11). Another way to deal with this problem is to correct the constant value of the quantum efficiency known at a reference temperature $T_{d r e f}$ with a law $f\left(\lambda, T_{d}\right)$ versus the wavelength and the temperature, as shown in (12)

$$
\eta_{e}\left(\lambda, T_{d}\right)=\eta_{e}\left(\lambda, T_{d r e f}\right) f\left(\lambda, T_{d}\right) .
$$

In NIR spectral band, the expression of $f$ is obtained from relation (11) and is defined by a macroscopic diffusion law, as follows:

$$
f=f_{2}\left(T_{d}\right)\left(\exp \left(f_{1}\left(\lambda, T_{d}\right)\right)+\exp \left(-f_{1}\left(\lambda, T_{d}\right)\right)\right)^{s i g n}
$$

where sign is equal to 1 when $\left(T_{d}-T_{d r e f}\right) \geq 0$ and -1 on the contrary, while $f_{1}$ is given by

$$
f_{1}\left(\lambda, T_{d}\right)=K_{1}\left(\lambda_{\text {norm }}\right)^{K_{2}}\left(T_{\text {dnorm }}\right)^{K_{3}}
$$

where:

- $\lambda_{\text {norm }}=\left(\lambda-\lambda_{\min }\right) /\left(\lambda_{\max }\left(T_{d}\right)-\lambda_{\min }\right), \lambda_{\max }$ and $\lambda_{\min }$ are respectively maximum and minimum wavelengths of NIR spectral band $\left(\lambda_{\max }=(h c) /\left(E_{g}\left(T_{d}\right) q\right)\right.$, $\left.\lambda_{\min }=0.75 \mu \mathrm{m}\right) . E_{g}$ is the energy gap $(\mathrm{eV})$ $\left(E_{g}=1.1557-\left(7.0210^{-4} T_{d}^{2}\right) /\left(1108+T_{d}\right)\right)($ see formula in [11]). 


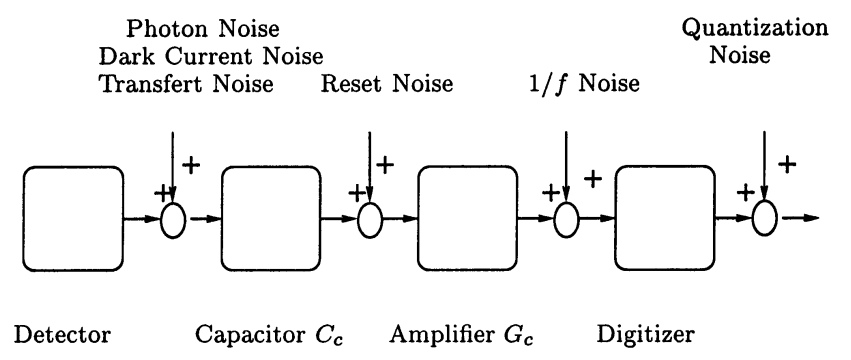

Fig. 4. Noise transfer diagram.

- $T_{\text {dnorm }}=\left|T_{d}-T_{\text {dref }}\right| /\left(T_{d \max }-T_{\text {dref }}\right), T_{\text {dmax }}$ is the maximal detector temperature.

Finally, $f_{2}$ is a constant $K_{4}$ raised to power of normalized detector temperature $T_{d n o r m}$. To obtain a ratio of 1 when $T_{d}=$ $T_{\text {dref }}$, we divide this term by 2 . We get

$$
f_{2}\left(T_{d}\right)=\frac{\left(K_{4}\right)^{T_{d n o r m}}}{2} .
$$

According to the first step of the determination of radiometric model, we compute the relation (12) at an effective wavelength $\lambda_{e}$

$$
\eta_{e}\left(\lambda_{e}, T_{d}\right)=\eta_{e}\left(\lambda_{e}, T_{d r e f}\right) f\left(\lambda_{e}, T_{d}\right)
$$

From model (16), the temperature-signal relation (7) is modified as follows:

$$
I_{D}=\frac{A_{3} \frac{G_{D} t i}{N^{2}} f\left(\lambda_{e}, T_{d}\right)}{\exp \left(\frac{B}{T}\right)-1}+D\left(T_{d}\right)
$$

where $A_{3}$ is a constant $\left(A_{3}=A_{2} \eta_{e}\left(\lambda_{e}, T_{d r e f}\right)\right)$.

\section{Coefficient D Model: Temporal Source Noise}

We model coefficient $D$ by the sum of a constant $I_{D O}$ and a variable $\overline{I_{D}}$ scaling the temperature $T_{d} . I_{D O}$ is associated with an offset and with noise sources independent of temperature. We assume that noise is independent of signal level and spatial variations. Therefore, we get

$$
D\left(T_{d}\right)=I_{D 0}+\overline{I_{D}}\left(T_{d}\right)
$$

Many books and articles (i.e., [12]), describe noise sources depending on the detector temperature. We have represented the main ones on Fig. 4.

The main source noise comes from dark current generated by thermal generation in silicon. It has been shown that the mean background dark current level increases with the temperature $T_{d}$ (Kelvin) and the integration time $t i(s)$, as follows:

$$
V_{c d a r k}=t i A_{\text {pix }}\left(A_{\text {dark }} T_{d}^{\beta}\right)^{\frac{1}{2}} \exp -\frac{E_{g}\left(T_{d}\right) q}{2 k T_{d}}
$$

where $A_{d a r k}$ and $\beta$ are constant ( $\beta=3$ in silicon device).

Considering zero as the mean value of the reset noise, we add to the model (19) a linear dependence with temperature which comes from a drift of electronic components. Then, the mean value of the digital output $\overline{I_{D}}$ fluctuates with temperature, as follows:

$$
\overline{I_{D}}=G_{D}\left(K_{5} t i T_{d}^{\frac{3}{2}} \exp -\frac{E_{g}\left(T_{d}\right) q}{2 k T_{d}}+K_{6} T_{d}\right)
$$

where $K_{5}$ and $K_{6}$ are constant $\left(K_{5}=\left(2_{n} / V_{c F R}\right) A_{\text {dark }}^{1 / 2}\right)$.

\section{Conclusion of Radiornetric Model Expression}

Finally, (21) describes our complete radiometric model with nine parameters $\left(A_{3}, B, I_{D O}, K_{1}, K_{2}, K_{3}, K_{4}, K_{5}\right.$, and $K_{6}$ ) which must be fitted during a calibration procedure

$$
I_{D}=\frac{A_{3} \frac{G_{D} t i}{N^{2}} f\left(\lambda_{e}, T_{d}\right)}{\exp \left(\frac{B}{T}\right)-1}+D\left(T_{d}\right) .
$$

where

$$
\begin{aligned}
f & =f_{2}\left(\exp \left(f_{1}\right)+\exp \left(-f_{1}\right)\right)^{\text {sign }} \\
f_{1} & =K_{1}\left(\lambda_{\text {norm }}\right)^{K_{2}}\left(T_{\text {dnorm }}\right)^{K_{3}} \\
f_{2} & =\frac{\left(K_{4}\right)^{T_{\text {dnorm }}}}{2} \\
D & =I_{D 0}+\overline{I_{D}}\left(T_{d}\right) \\
I_{D 0} & =\text { constant } \\
\overline{I_{D}} & =G_{D}\left(K_{5} t i T_{d}^{\frac{3}{2}} \exp -\frac{E_{g}\left(T_{d}\right) q}{2 k T_{d}}+K_{6} T_{d}\right) .
\end{aligned}
$$

\section{E. Radiometric Model Calibration}

First, from the CCD camera model (21), we estimate the dependence of the output signal noise fl function of the detector temperature to fix the $I_{D O}, K_{5}$, and $K_{6}$ parameters. Then, to fit missing parameters, the CCD camera has been calibrated using blackbody temperatures from 320 to $460{ }^{\circ} \mathrm{C}$ intervals with detector temperature variation range $-30+52{ }^{\circ} \mathrm{C}$.

On our experiments, we have tested several kinds of CCD cameras but we present only the results for the VHR 2000 CCD camera manufactured by Digital Vision. The CCD is a Sony $I C X 039752(H) \times 582(V)$ array of $8.6(H) \times 8.3(V) \mu \mathrm{m}^{2}$ pixels. This camera has a linear response with exposure time. We add Kodak Wratten 87C near infrared selected filter. All experimental tests have taken place in a dark environment where the detector temperature $T_{d}$ was regulated with an air-conditioned enclosure.

1) Observations of System Noise: In a dark environment and when the detector temperature $T_{d}$ is adjusted between -30 and $+60{ }^{\circ} \mathrm{C}$, we compute the mean value $D$ of the digital image. Considering an exposure time $t i$ of $20 \mathrm{~ms}$ and a unitary gain $G_{D}$, Fig. 5 shows a linear evolution for $D$ until temperature reaches $20{ }^{\circ} \mathrm{C}$. Above this temperature, $D$ increases exponentially mainly because of current noise. By fitting equation (18) to these $D$ values, we have deduced $I_{D O}, K_{5}$, and $K_{6}$ parameters which are provided in Table I.

This model allows to estimate correctly the noise fluctuations for our uncooled CCD camera. Note that long exposure time requires a frame integration time concept. Charges are collected simultaneously but are read out alternately. Changing the gate voltage shifts the image centroid by half a pixel in vertical direction. This creates $50 \%$ overlap and therefore pixel sensitivity is doubled. This effect leads to an average of the noise. 


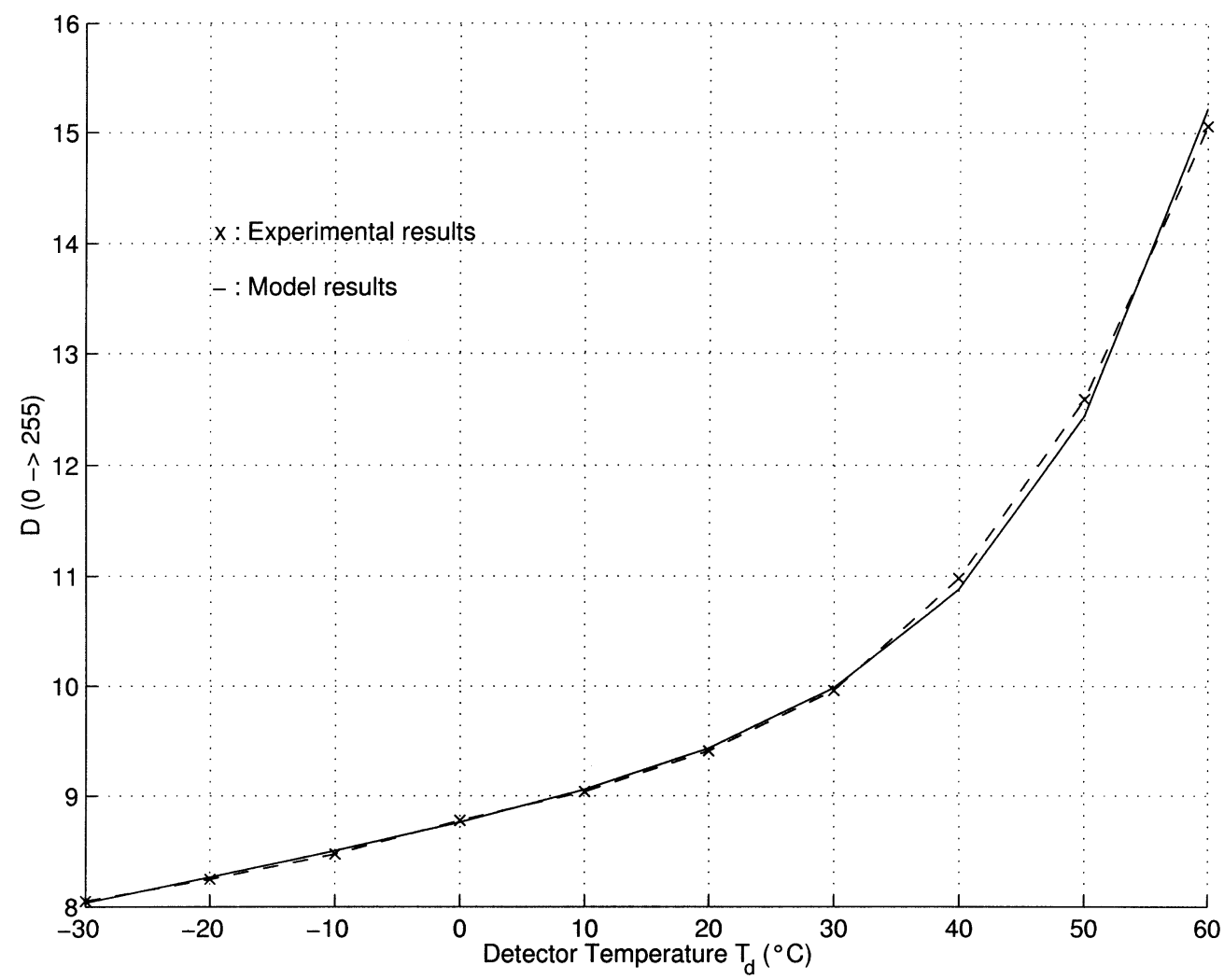

Fig. 5. $D$ as a function of $T_{d}$.

TABLE I

PARAMETERS VALUES OF MODEL (18)

\begin{tabular}{c|c|c}
\hline$I_{D 0}$ & $K_{5}$ & $K_{6}$ \\
\hline 2.41 & $1.79410^{+5}$ & $2.3110^{-2}$ \\
\hline
\end{tabular}

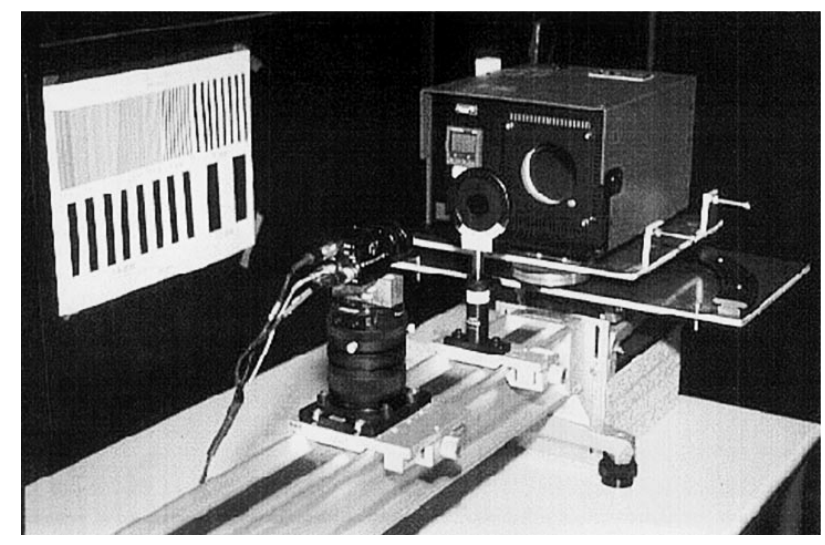

Fig. 6. Experiment for radiometric calibration.

2) Temperature Calibration: In order to estimate the missing parameters of model (21), we have directly illuminated the camera by a nearly spatially uniform blackbody (see Fig. 6). In order to cover a maximum of pixels, we have used a blackbody with a large cavity (64-mm aperture). The temperature range is from 20 to $550{ }^{\circ} \mathrm{C}$. For a focal length $f$ of $8 \mathrm{~mm}$, a F-number $\mathrm{N}$ of 1.4, an integration time $t i$ of $360 \mathrm{~ms}$, and an output amplifier gain $G_{D}$ of 1.41 , we have computed the mean

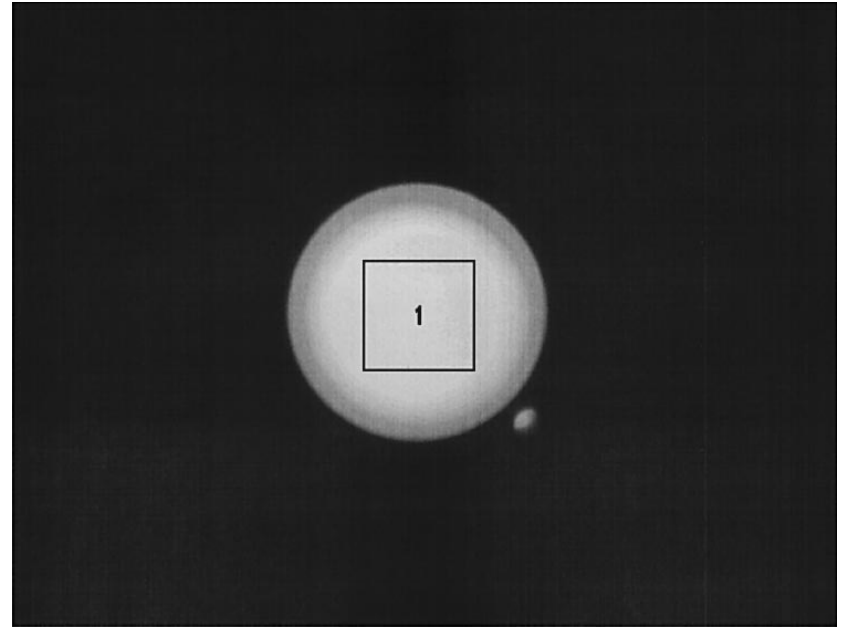

Fig. 7. (1) Black-body uniform region.

value $\left(I_{D}\right)$ over a number of repeated image central regions (see Fig. 7). Within these experimental conditions, a value of $I_{D}$ over the range [0-255 Gray Levels] involves a black-body temperature range from 300 to $460{ }^{\circ} \mathrm{C}$. Of course, with a lower time exposure value, it is possible to increase the blackbody temperature without signal saturation.

Using these experimental points $\left(T, I_{D}\right)$, the calibration procedure to estimate the $A_{3}, B, K_{1}, K_{2}, K_{3}$, and $K_{4}$ parameters of model (21) is as follows.

1) For the reference detector temperature $T_{\text {dref }}: A_{3}$ and $B$ determination. 


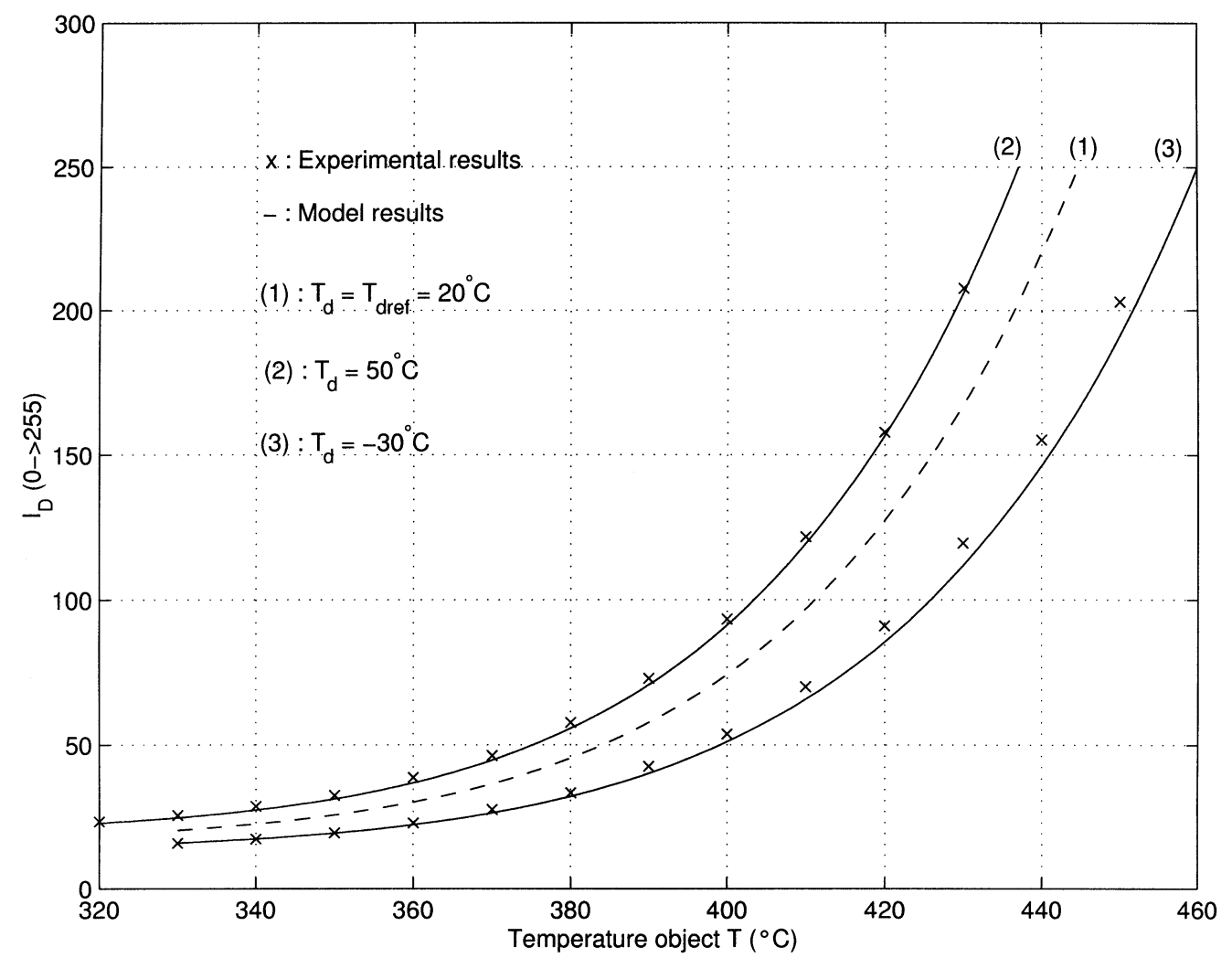

Fig. 8. Calibration and verification curves for different $T_{d}$.

Usually, the manufacturer gives data at a reference temperature $T_{\text {dre } f}$ of $20{ }^{\circ} \mathrm{C}$. In this case, the correction function $f\left(\lambda_{e}, T_{d}\right)$ of the detector temperature is a unitary function in model (21). Curve 1 of Fig. 8 shows experimental points and model with $A_{3}$ and $B$ estimated parameters.

2) For a different temperature $T_{d}: K_{1}, K_{2}, K_{3}$, and $K_{4}$ determination.

With a temperature different from $T_{d r e f}$, knowing $A_{3}$ and $B$ parameters, we can estimate the parameters of the function $f\left(\lambda_{e}, T_{d}\right)$. The results of experiment and estimation procedure obtained with a detector temperature $T_{d}$ of $50{ }^{\circ} \mathrm{C}$.

3) Experimental verification of the model (21).

Considering the previous fitted parameters of model (21) and for a different value $T_{d}$ of $-30{ }^{\circ} \mathrm{C}$, curve (3) of Fig. 8 represents experimental and computed results. The difference between experimental and calculated curve is lower than $1 \%$ of object temperature. Including all uncertainties, the model (21) with fitted parameters provided in Table II can be accepted.

\section{F. Performances}

Our experiments have taken into account detector temperature variations in the radiometric model (21) over a range from -30 to $50{ }^{\circ} \mathrm{C}$. We model effects related to detector temperature in quantum efficiency due to variation in light absorption. In the reference of absorption coefficient formulas [13], the model can
TABLE II

PARAMETERS VALUES OF MODEL (21)

\begin{tabular}{c|c|c|c|c|c}
\hline$A_{3}$ & $B$ & $K_{1}$ & $K_{2}$ & $K_{3}$ & $K_{4}$ \\
\hline $1.01110^{+12}$ & $1.50510^{+4}$ & 1.743 & 1.396 & 0.631 & 1.018 \\
\hline
\end{tabular}

be extended to detector temperature lower than $-30{ }^{\circ} \mathrm{C}$ down to $-50{ }^{\circ} \mathrm{C}$. At the other end, for temperatures higher than $50{ }^{\circ} \mathrm{C}$ up to $80{ }^{\circ} \mathrm{C}$ (CCD detector is still operating), no experiment shows the validity of the model. However, we can observe a high level of dark current (see Fig. 5). Knowing the detector temperature with an external sensor, the radiometric model (21) allows object temperature correction of $25^{\circ} \mathrm{C}$ for a gray level value of 100 (see Fig. 8).

Particularly, in a measurement context, it is important to qualify the radiometric model performances in terms of signal-to-noise ratio (SNR) and noise equivalent temperature difference (NETD) as functions of detector temperature $T_{d}$. Our goal described in the introduction, is to measure a low object temperature $T$ of $350{ }^{\circ} \mathrm{C}$ with uncertainty value of $\pm 25^{\circ} \mathrm{C}$. At this object temperature, Fig. 9 illustrates the SNR as a function of the temperature detector $T_{d}$ for different exposure time. The signal is provided by model (21) and the square root of the noise variance comes from the model (18). We observe a maximum SNR at a detector temperature $T_{d}$ of $18{ }^{\circ} \mathrm{C}$. Below this temperature value, the quantum efficiency limits the SNR value. At high detector temperature, dark current dominates and the SNR decreases. The NETD criterion is defined as the ratio of the standard deviation noise to the sensitivity that 


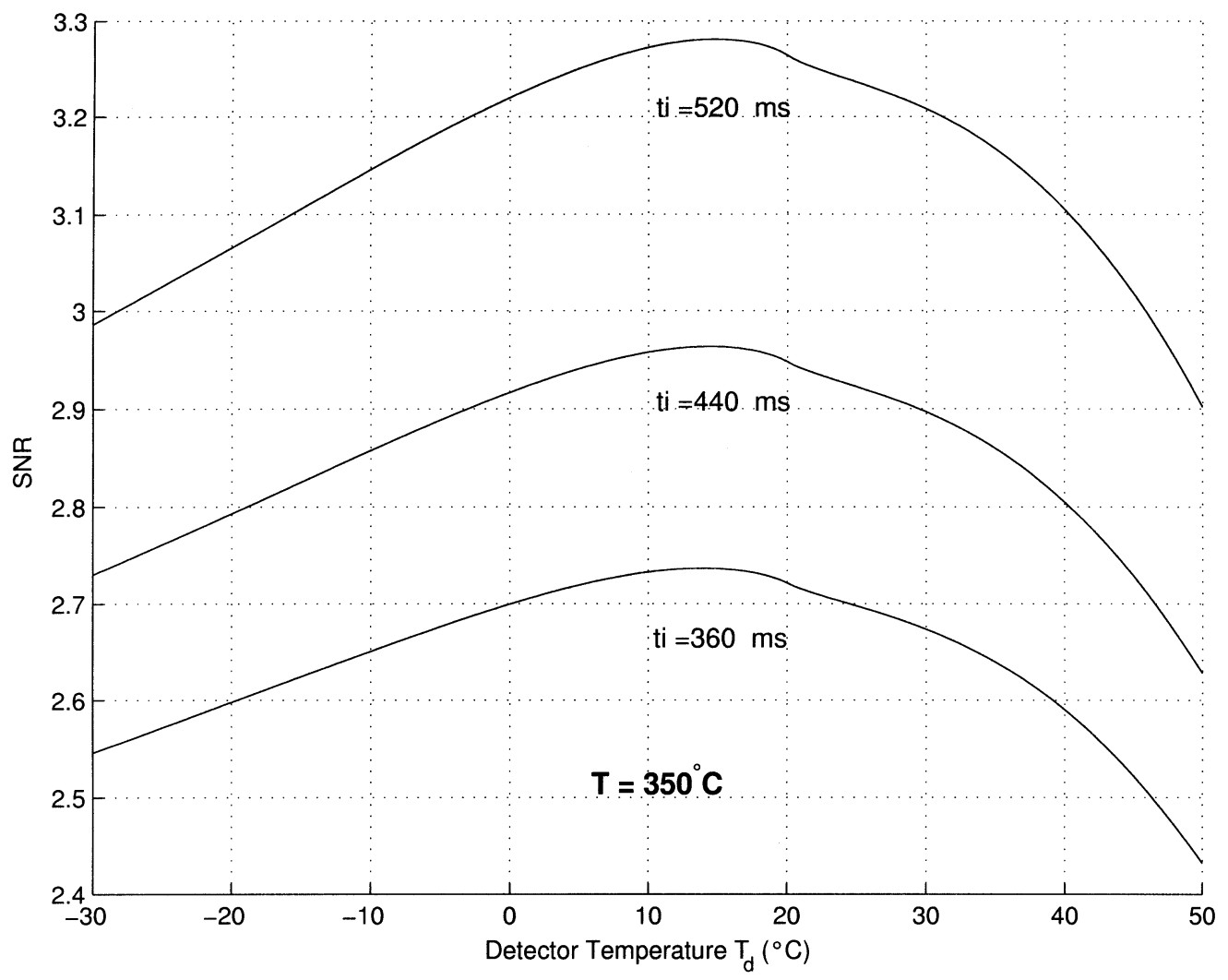

Fig. 9. SNR as function of $T_{d}$.

represents the derivative radiometric model (21) in function of object temperature $T$. We compute it for an object temperature $T_{0}$ of $350{ }^{\circ} \mathrm{C}$. The result of NETD versus temperature and for different exposure time is plotted in Fig. 10. In the same way, NETD curves present also a minimum at detector temperature of $18{ }^{\circ} \mathrm{C}$. For a low detector temperature, sensitivity increases more than noise. On the contrary, for a high detector temperature, noise dominates.

As a conclusion, high detector temperatures degrade camera performances, but it is possible to adjust exposure time $t i$ to respect values of SNR or NETD. For example, at reference detector temperature, to measure over the range from 350 to $1000{ }^{\circ} \mathrm{C}$ with SNR higher than 2 and an NETD value always higher than $8{ }^{\circ} \mathrm{C}$, five exposure times are required over the range from 0.36 s to $1 / 10000 \mathrm{~s}$. The value of F-number $N$ and amplifier output gain $G_{D}$ are the same as those used in experiments.

\section{GEOMETRIC MODEL}

This section describes the geometric model of the imaging system (optical lens, camera, and digital card). After an overview of the "pin-hole" model used in our applications, we define their extrinsic and intrinsic parameters to be calibrated. Extrinsic parameters describe the transformation of the object 3-D frame into the camera 3-D frame. The transformation of the latter frame into the image two-dimensional (2-D) frame is expressed by intrinsic parameters. They depend on optic perspective projection and detector CCD spatial resolution. By studying the MTF, we examine the influence of spectral band and detector temperature on the spatial resolution. This analysis shows that intrinsic parameters depend on spectral band and detector temperature. In this section, we do not discuss the accuracy of intrinsic parameters in function of 3-D error models or 2-D errors introduced by the target detection in the camera image [14].

\section{A. "Pin-Hole" Model}

The basic assumption of the "pin-hole" model is that the lens center $O_{\text {cam }}$ is the intersection of all optical beams. We assume that the image plane (or CCD detector plane) is perpendicular to optical axis $\left(O_{c a m}-z\right)$. Fig. 11 illustrates the basic geometry of the pin-hole camera model. $R_{o b j}\left(0_{o b j}, \vec{X}, \vec{Y}, \vec{Z}\right)$ represents the object $3-\mathrm{D}$ frame, while $R_{\text {cam }}\left(0_{\text {cam }}, \vec{x}, \vec{y}, \vec{z}\right)$ denotes the camera 3-D frame and $R_{i m}\left(0_{i m}, \vec{u}, \vec{v}\right)$ is the image 2-D frame. Finally, $\left(u_{0}, v_{0}\right)$ is the principal point, or the optical center $\left(0_{\text {cam }}\right)$ projection on the CCD detector plane.

Three steps are required to compute the transformation from a point $(X, Y, Z)$ in $R_{o b j}$ to a point $p^{\prime}(u, v)$ in $R_{i m}$.

1) Rigid body transformation (rotation $R$ and translation $T$ ) of the object coordinates $(X, Y, Z)$ in $R_{o b j}$ to camera coordinates $(x, y, z)$ in $R_{c a m}$.

2) Perspective projection with center $0_{\text {cam }}$, axis $\vec{z}$ and ratio $f$ (distance between the CCD detector and the optical center), of the 3 -D point $(x, y, z)$ into an image point $(i$, $j, f)$ expressed in the camera 3-D frame.

3) Transformation of camera coordinates $(i, j, f)$ into 2-D image frame coordinates $(u, v)$ using an origin transformation $\left(u_{0}, v_{0}\right)$ in image plane and a space sampling ac- 


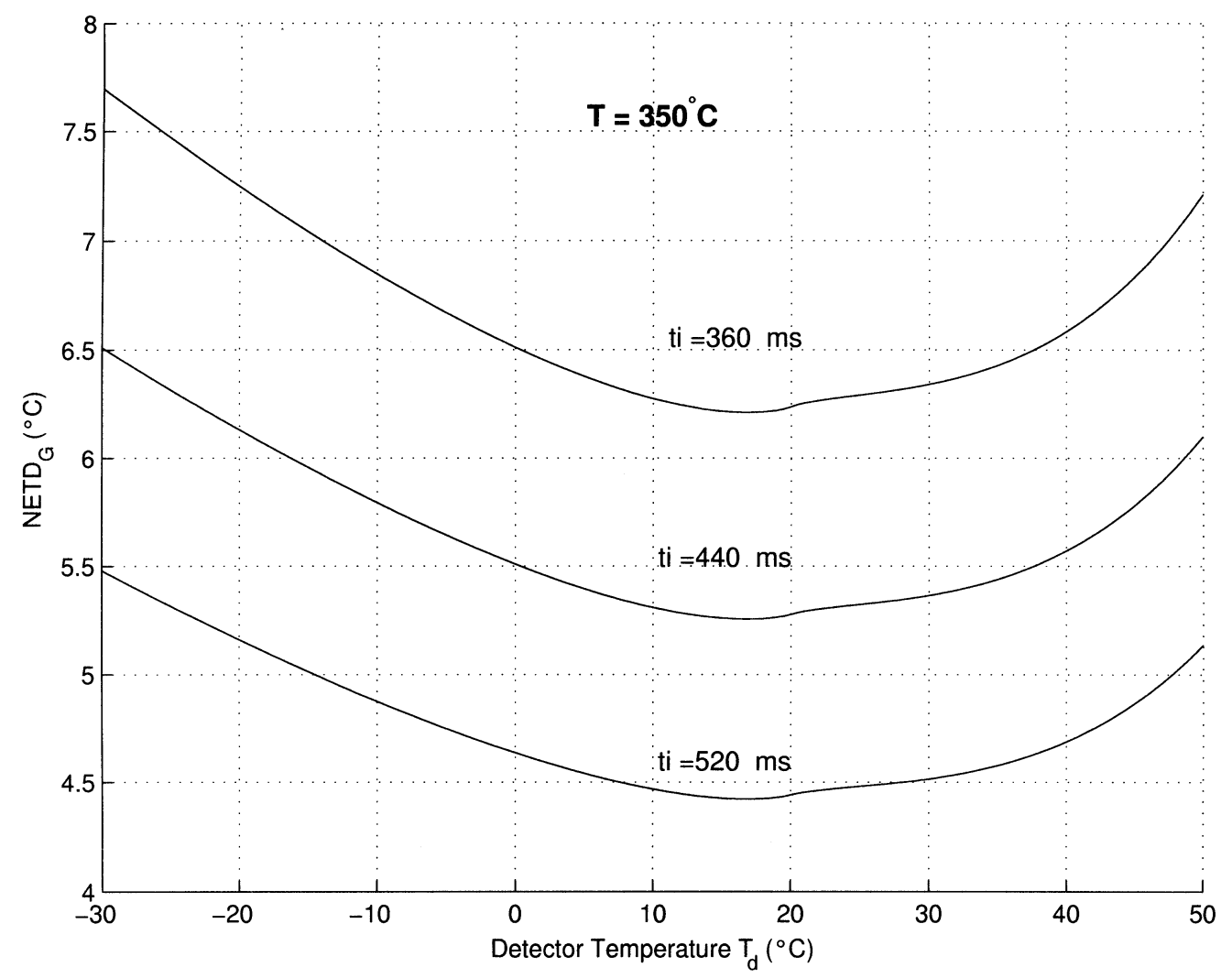

Fig. 10. NETD as function of $T_{d}$.

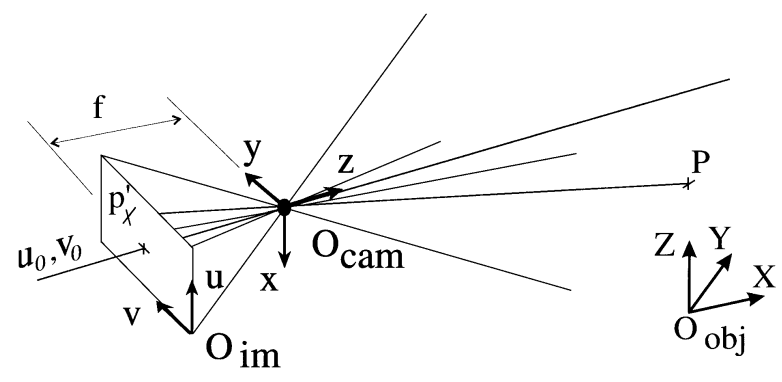

Fig. 11. Object and camera coordinate system.

cording to the pixel sizes $\Delta x$ and $\Delta y$ (considering pixels as adjacent in CCD array). The dependence of pixel sizes on wavelength and detector temperature will be discussed in paragraph III-B.

By combining these three steps, (22) relates the transformation of the 3-D object into the 2-D image frame

$$
\left(\begin{array}{c}
u \\
v
\end{array}\right)=\left(\begin{array}{c}
u_{0} \\
v_{0}
\end{array}\right)+\left(\begin{array}{l}
\frac{f}{\Delta x\left(\lambda, T_{d}\right)} \frac{R_{11} X+R_{12} Y+R_{13} Z+T_{x}}{R_{31} X+R_{32} Y+R_{33} Z+T_{z}} \\
\frac{f}{\Delta y\left(\lambda, T_{d}\right)} \frac{R_{21} X+R_{22} Y+R_{23} Z+T_{y}}{R_{31} X+R_{32} Y+R_{33} Z+T_{z}}
\end{array}\right) .
$$

Model (22) involves 12 extrinsic parameters (nine for rotation $R$ and three for translation $T$ ) and four intrinsic parameters [ $u_{0}$, $\left.\left.v_{0}, \alpha_{u}=\left(f / \Delta x\left(\lambda, T_{d}\right)\right), \alpha_{v}=\left(f / \Delta_{y}\left(\lambda, T_{d}\right)\right)\right)\right]$. To simplify the presentation, we do not introduce in this paper the lens distortion correction step described in [15]. We use this correction in our applications.

The model calibration described in [8] or [16] involves a computation of the camera extrinsic and intrinsic parameters based

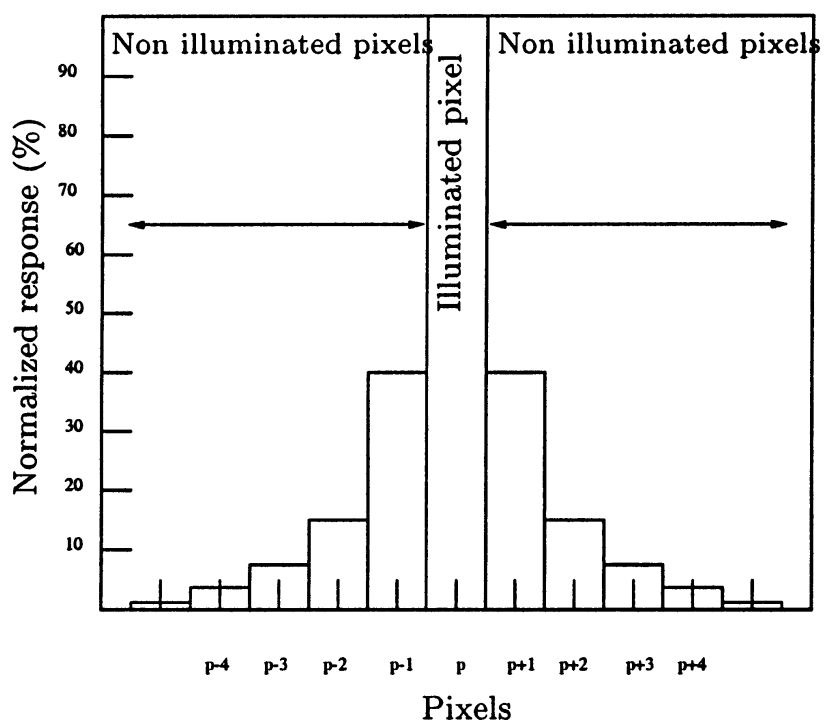

Fig. 12. Diffusion influence.

on several points which object coordinates $(X, Y, Z)$ in the 3-D object frame $R_{o b j}$ are known and which image coordinates $(u, v)$ are measured. These points are extracted from several images of a specific object (planar calibration pattern), moved in front of the camera.

For robotic applications, self-calibration methods [17] have been proposed to recover these parameters on line, using a weak camera model. For our measurement applications, we apply a strong calibration method, which requires two steps: a) using 


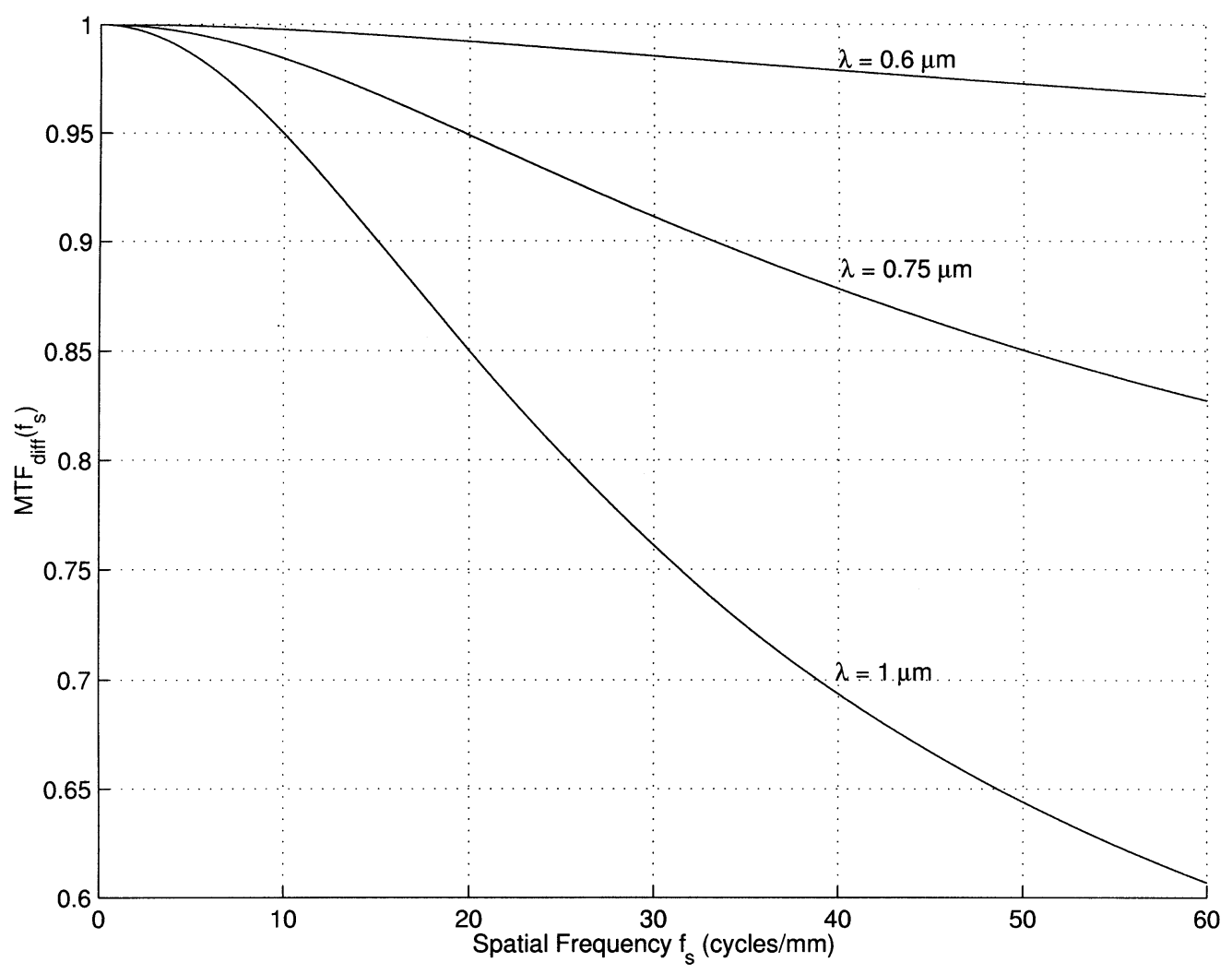

Fig. 13. $\operatorname{MTF}_{\text {diff }}$ as a function of $\lambda$.

initial estimates of the intrinsic parameters computed from the camera characteristics, the object pose computation [18] gives estimates for the extrinsic parameters for each image acquired on the calibration object. b) Then, from the initial guesses, a non linear minimization method (including the estimation of the distortion coefficients) allows to improve the estimation of these parameters. A basic hypothesis for this procedure is that focal length $f$ and pixel sizes $\Delta x$ et $\Delta y$ remain constant. If it is not the case (active camera or fluctuations according to the wavelength and to the detector temperature), they must be estimated again.

The metrology applications require very accurate estimates of the camera parameters: therefore we have to verify if intrinsic parameters, especially $\alpha_{u}=f / \Delta x$ and $\alpha_{v}=f / \Delta y$, are changing with spectral band and detector temperature fluctuations. The MTF of the camera characterizes the optic effects and how the discrete locations of detector elements $(\Delta x, \Delta y)$ sample spatially the scene in function of wavelength and detector temperature.

\section{B. Camera MTF}

The complete MTF of the camera is the product of the optic $\operatorname{MTF}\left(\mathrm{MTF}_{o}\right)$ and the detector $\mathrm{MTF}\left(\mathrm{MTF}_{d}\right)$, that is

$$
\mathrm{MTF}=\mathrm{MTF}_{o} \times \mathrm{MTF}_{d}
$$

1) Optic $M T F_{o}$ : With reference to [19], $\mathrm{MTF}_{o}$ in the horizontal or vertical direction of a radial symmetric optical system with a clear circular diffraction-limited aperture illuminated monochromatically is given by

$$
\begin{aligned}
\operatorname{MTF}_{o}\left(f_{s}\right)= & \frac{2}{\pi}\left(\cos ^{-1}\left(\frac{f_{s}}{f_{o c}}\right)\right. \\
& \left.-\left(\frac{f_{s}}{f_{o c}}\right) \sqrt{\left(1-\left(\frac{f_{s}}{f_{o c}}\right)^{2}\right)}\right)
\end{aligned}
$$

where $f_{o c}$ is the cutoff frequency of the optical system $\left(f_{o c}=\right.$ $(1 / N \lambda)$. In NIR spectral band, with an F-number $N$ equal to $1.4, \mathrm{MTF}_{o}$ is close to $1(0.998-0.978)$. The camera MTF can then be approximated by $\mathrm{MTF}_{d}$

$$
\mathrm{MTF} \approx \mathrm{MTF}_{d}
$$

The camera cutoff frequency $f_{c}$ is equal to the detector cutoff frequency $f_{d c}$.

2) Detector $M T F_{d}:$ CCD detector is a spatial sampler of a horizontal frequency $f_{H s}$ (respectively vertical $f_{V s}$ ) of the input signal. The highest CCD horizontal frequency reproduced is the Nyquist horizontal frequency $f_{H N}$ (respectively vertical $f_{V N}$ ). It is mandatory that the input signal frequency are inferior to Nyquist frequency to respect Shannon theorem and to avoid spatial aliasing. In metrology applications, the Nyquist frequency is the band-limit or the system cutoff frequencies $f_{d c}$ which is defined as half an inverse pixel dimension: $f_{H N}=(1 / 2 \Delta x)$ [respectively, $f_{V N}=(1 / 2 \Delta y)$ ]. We assume here that pixels are 


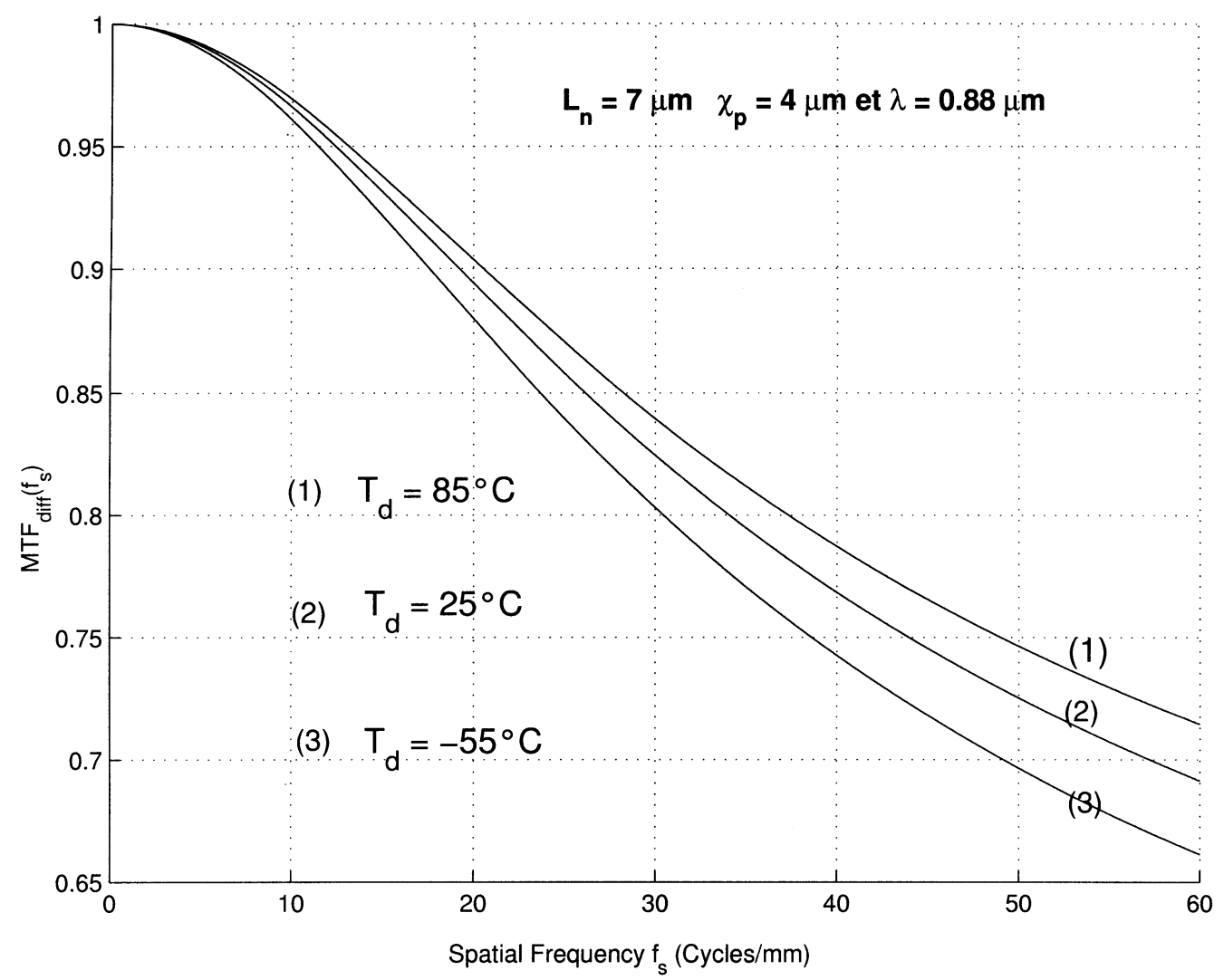

Fig. 14. $\mathrm{MTF}_{\text {diff }}$ as a function of $T_{d}$.

adjacent. We note that the detector cutoff frequency decreases when the cell area increases.

Moreover of the influence of the detector geometry, in NIR spectral band, the photons absorption occurs at increasing depths along substrate detector depth $z$. The absorption law $\Phi$ approaches an exponential law $\left[\left(\Phi=C^{t} \exp (-\alpha z)\right)\right]$ where the coefficient absorption $\alpha$ in the silicon decreases with an increasing wavelength (see Fig. 3). However, the depletion region size $Z D$ is finite (see Fig. 2) and the majority of long wavelength photons will be absorbed outside of the depletion region. An electron generated in substrate will experience a 3-D random walk until it recombines or reaches the edge of neighbor depletion region. In the last case, this phenomenon, called diffusion, creates a response that overlaps other pixels (see Fig. 12 in reference of [13]).

Finally, in NIR spectral band, the detector MTF $\left(\mathrm{MTF}_{d}\right)$ results of the product of the geometric MTF $\left(\mathrm{MTF}_{\text {geo }}\right)$ by the diffusion MTF $\left(\mathrm{MTF}_{\text {diff }}\right)$, and it is expressed by

$$
\mathrm{MTF}_{d}=\mathrm{MTF}_{g e o} \times \mathrm{MTF}_{d i f f} .
$$

According to [20], the geometric $\mathrm{MTF}_{g e o}$ of a single CCD cell is given by

$$
\operatorname{MTF}_{g e o}\left(f_{H s}, f_{V s}\right)=\left|\operatorname{sinc}\left(\pi \frac{f_{H s}}{2 f_{H N}}\right)\right|\left|\operatorname{sinc}\left(\pi \frac{f_{V s}}{2 f_{V N}}\right)\right| .
$$

In order to simplify the expression, we compute the $\mathrm{MTF}_{\text {geo }}$ only in one dimension for the horizontal direction $x$. Therefore, we denote the horizontal input frequency $f_{s H}$ as $f_{s}$ and horizontal Nyquist frequency $f_{H N}$ as $f_{N}$. For $f_{N}=f_{d c}=f_{s}$, the $\mathrm{MTF}_{\text {geo }}$ value is 0.637 .

For a front illuminated CCD, the diffusion term $\mathrm{MTF}_{\text {diff }}$ in reference of [21] can be written as

$$
\operatorname{MTF}_{d i f f}\left(\lambda, T_{d}\right)=\frac{1-\frac{\exp \left(-\alpha\left(\lambda, T_{d}\right) \mathcal{X}_{p}\right)}{1+\alpha\left(\lambda, T_{d}\right) L_{o}\left(f_{s}\right)}}{1-\frac{\exp \left(-\alpha\left(\lambda, T_{d}\right) \mathcal{X}_{p}\right)}{1+\alpha\left(\lambda, T_{d}\right) L_{n}}}
$$

where the factor $L_{o}$ is the spatial frequency-dependent component of diffusion length $L_{n}\left(L_{o}=L_{n} /\left(\sqrt{1+\left(2 \pi L_{n} f_{s}\right)^{2}}\right)\right.$ and $\chi_{p}$ is the depletion width (see Fig. 2).

Let us remark that the diffusion term $\mathrm{MTF}_{\text {diff }}$ depends on the absorption coefficient as a function of wavelength and temperature as shown in Fig. 3.

Fig. 13 shows MTF $_{\text {diff }}$ versus wavelength $\left(\chi_{p}=4 \mu \mathrm{m}\right.$, $\left.L_{n}=7.1 \mu \mathrm{m}\right)$. For short wavelengths $(\lambda<0.6 \mu \mathrm{m}), \mathrm{MTF}_{\text {diff }}$ can be neglected and it approaches $1\left(\mathrm{MTF}_{d} \approx \mathrm{MTF}_{g e o}\right)$. For the NIR spectral band $(\lambda>0.75 \mu) \mathrm{m}$, the diffusion term

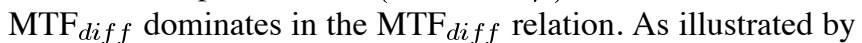
Fig. 14, the diffusion term $\mathrm{MTF}_{\text {diff }}$ decreases with the detector temperature.

As a conclusion, in visible spectral band, we can approximate the camera MTF with only the geometric MTF $\left(\mathrm{MTF}_{g e o}\right)$ of the detector

$$
\mathrm{MTF}=\mathrm{MTF}_{d}=\mathrm{MTF}_{g e o} .
$$




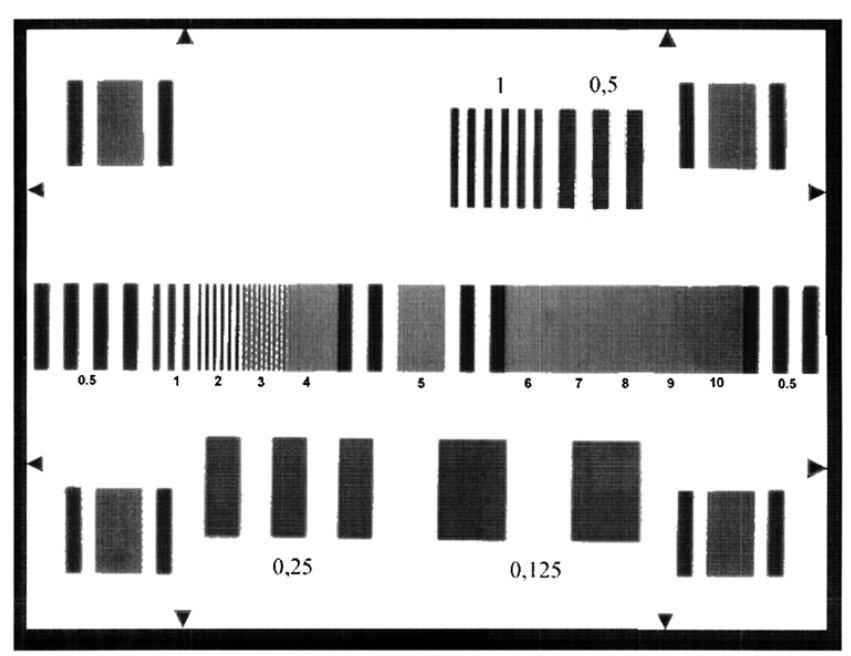

Fig. 15. Bar test target.

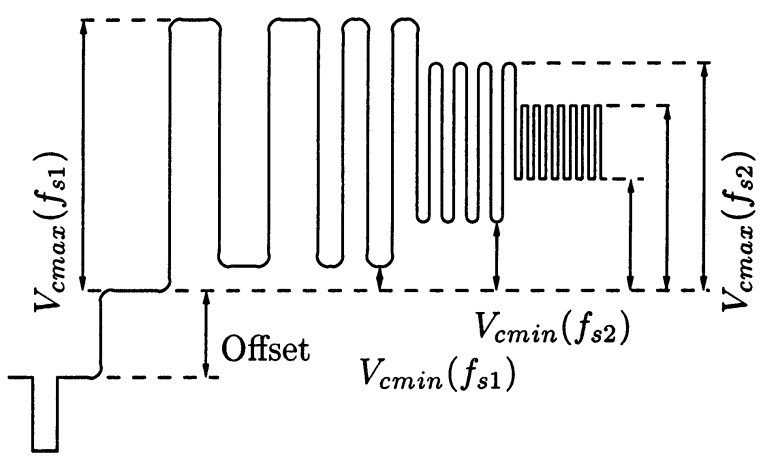

Fig. 16. Definition of $V_{c m a z}$ and $V_{c m i n}$.

But, in NIR spectral band, the cameraMTF must be expressed as the product of the geometric MTF $\left(\mathrm{MTF}_{g e o}\right)$ by the diffusion $\operatorname{MTF}\left(\mathrm{MTF}_{d i f f}\right)$

$$
\mathrm{MTF}=\mathrm{MTF}_{d}=\mathrm{MTF}_{g e o} \times \mathrm{MTF}_{d i f f} .
$$

With the same value of MTF $(\mathrm{MTF}=0.637)$, the camera cutoff frequency $f_{c}$ is lower in NIR than in visible spectral band. In addition, its value decreases with the detector temperature. Finally, the pixel effective sizes $\left(\Delta x=1 / 2 f_{c}\right)$ are higher in NIR than in visible spectral band.

\section{Experimental Results}

Although the camera MTF is defined for sinusoidal signals, the square wave (see Fig. 15) is the most popular test target. The system response to a square-wave target is the contrast transfer function $(\mathrm{CTF})$

$$
\operatorname{CTF}\left(f_{s}\right)=\frac{V_{c \max }-V_{c \min }}{V_{c \max }+V_{c \min }}
$$

where $V_{c \max }$ and $V_{c \min }$ are respectively maximum and minimum signal levels of white and black bar test (see Fig. 16). To obtain the relationship between square wave and sinusoidal amplitude, we have to express the square wave as cosine series. The output amplitude is an infinite sum of input cosine amplitudes modified by MTF

$$
\operatorname{CTF}\left(f_{s 0}\right)=\frac{4}{\pi}\left|\operatorname{MTF}\left(f_{s 0}\right)-\frac{\operatorname{MTF}\left(3 f_{s 0}\right)}{3}+\cdots\right|
$$

where $f_{s 0}$ represents the fundamental frequency of the square wave.

For bar target with spatial frequency $f_{s}$ above $1 / 3$ of frequency computed to $\mathrm{MTF}=0$, we can write the camera MTF from the measured $\mathrm{CTF}$, as follows:

$$
\mathrm{MTF}=\frac{\pi}{4} \mathrm{CTF} .
$$

After measuring the CTF of the VHR 2000 CCD camera tested in paragraph II-E, from (33) we compute the MTF and we compare the cutoff frequency $f_{c}$ (for a value of 0.637 of the MTF) in visible and in NIR spectral band. We also examine the influence of the detector temperature on the MTF.

1) MTF as a Function of Wavelength: Using experimental and model results (MTF, $f_{s}$ ) plotted in Fig. 17, we can compute the cutoff frequency $f_{c}$, as follows.

- In visible spectral band $\left(\mathrm{MTF}_{\text {diff }} \approx 1\right)$ :

For a value of 0.637 of the MTF, the value of the cutoff frequency $f_{c}$ is $45.3 \mathrm{~mm}^{-1}$. From relation $\left(\Delta x=1 / 2 f_{c}\right)$, it easily follows that the value of the detector width $\Delta x$ is $11 \mu \mathrm{m}$. The value is different from the manufacturer's $(\Delta x=8.6 \mu \mathrm{m}$ due to a low pass filter with a cutoff frequency lower than Nyquist frequency to satisfy the Shannon sampling theorem.

- In NIR spectral band:

Considering experimental results plotted in Fig. 17, MTF produced in NIR is lower than MTF produced in visible spectral band. We can estimate $L_{n}$ and $\chi_{p}$ parameters of the diffusion term $\mathrm{MTF}_{\text {diff }}$ (28) in the model (30). The values are provided in Table III. As a result, the value of the cutoff frequency $f_{c}$ is $30.50 \mathrm{~mm}^{-1}$ leads to a detector width value $\Delta x$ of $16.39 \mu \mathrm{m}$.

We note that the diffusion length value $L_{n}$ is small. The limited diffusion in substrate can be explained at least in part by a likely epitaxial layer. This fact can be also ascribed to an inadequate 2-D diffusion model to explain 3-D diffusion mechanisms.

As a conclusion, in NIR spectral, MTF decreases and it is equivalent to work with detector sizes 1.34 times higher.

2) MTF as a function of the detector temperature: To take into account the effects of the detector temperature, considering previous values of $L_{n}$ and $\chi_{p}$ parameters (see Table III), we compute the absorption coefficient $\alpha$ (see formulae in Appendix) as a function of detector temperature $T_{d}$ ranging from -50 to $80{ }^{\circ} \mathrm{C}$. From these computations and the model (30) in NIR spectral band, we obtain the MTF values versus temperature $T_{d}$. For MTF value of 0.637 , we can deduce the cutoff frequency $f_{c}$ and compute the detector width $\left(\Delta x=1 / 2 f_{c}\right)$ as a function of $T_{d}$. The Fig. 18 shows a linear decreasing of the detector width $\Delta x$ with a detector temperature increasing $T_{d}$. We can infer the following linear model:

$$
\Delta_{x}\left(T_{d}\right)=\Delta_{x}\left(T_{d r e f}\right)-K_{7}\left(T_{d}-T_{d r e f}\right)
$$




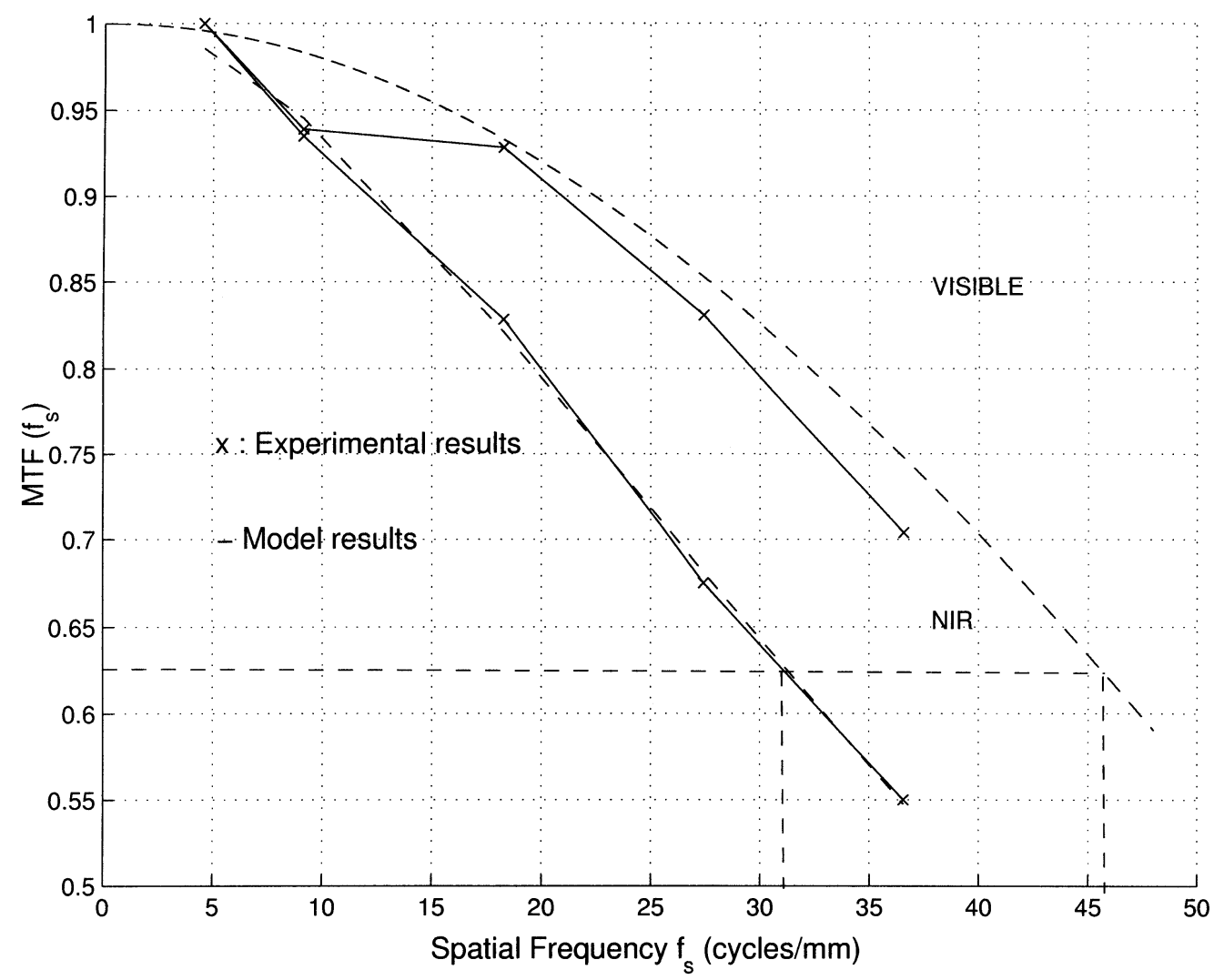

Fig. 17. Experimental verification of MTF.

TABLE III

PARAMETER VALUES OF MODEL (28)

\begin{tabular}{c|c}
\hline$L_{n}(\mu m)$ & $\chi_{p}(\mu m)$ \\
\hline 7.101 & 3.36 \\
\hline
\end{tabular}

where $K_{7}$ is a parameter which value $\left(K_{7}=7.23510^{-3}\right)$ has been obtained from the experimental points plotted in Fig. 18.

\section{Performances}

By studying the MTF as a function of wavelength, we notice that the spatial resolution of the CCD detector decreases with an increasing of wavelength to account for diffusion phenomenon. To fit the intrinsic parameters of the "pin-hole" camera model, the system has been calibrated in NIR spectral band.

The same studies show an increasing of spatial resolution with detector temperature. We propose a correction law (34) of intrinsic parameters $\alpha_{u}$, and $\alpha_{v}$ as a function of detector temperature $T_{d}$. With a limited substrate detector by an epitaxial layer, the correction is less necessary than with a thick substrate detector (for example with the detector Hamamatsu S5466). The model calibration has been tested in visible and in NIR spectral band with test points extracted from lozenge center. We estimate intrinsic parameters with method [16] and we obtain $\alpha_{u}$ and $\alpha_{v}, 1131.17,1037.34$ in visible and, respectively, 1139.53 and 1045.09 in NIR spectral band. The localization error given by method described in is $0.1 \mathrm{~mm}$ at an observation distance of $1 \mathrm{~m}$. This experiment should be conducted many times to verify parameters dispersion.
IV. Two MODELS INTERESTS

In the two previous sections, we have presented radiometric and geometric calibration procedures for an uncooled CCD camera. We emphasize on the fluctuations of the camera parameters with respect to the detector temperature and to the wavelength.

In this section, we show the interest of these two models in order to improve some measurements on a hot object, which can be in any situation in the view field of the camera. In fact, variable orientation $\theta_{s}$ and distance $d_{s}$ of a hot object could be estimated by a 3-D localization. For monocular vision (only one camera), it could be impossible to locate a single hot point. This point must be associated to a surface belonging to an object. The object position with respect to the camera, can be computed only if the 3-D geometric model of the object has been previously learnt. For an application concerning the monitoring of free-form objects, a rigid visual pattern can be fixed on the object to make this localization easier by using a classical method [8]. For multiocular vision (passive stereo vision), a hot point could be detected and localized using triangulation methods. But, it is required that this point is in the view field of the two cameras. A first estimate on the radiometric attributes, computed without any knowledge on the orientation and the distance, could be used as a stereo-matching criterion. Then, once the point distance is known, the orientation could be computed from local planar approximations, and the radiometric attributes could be refined using the equation (36). 


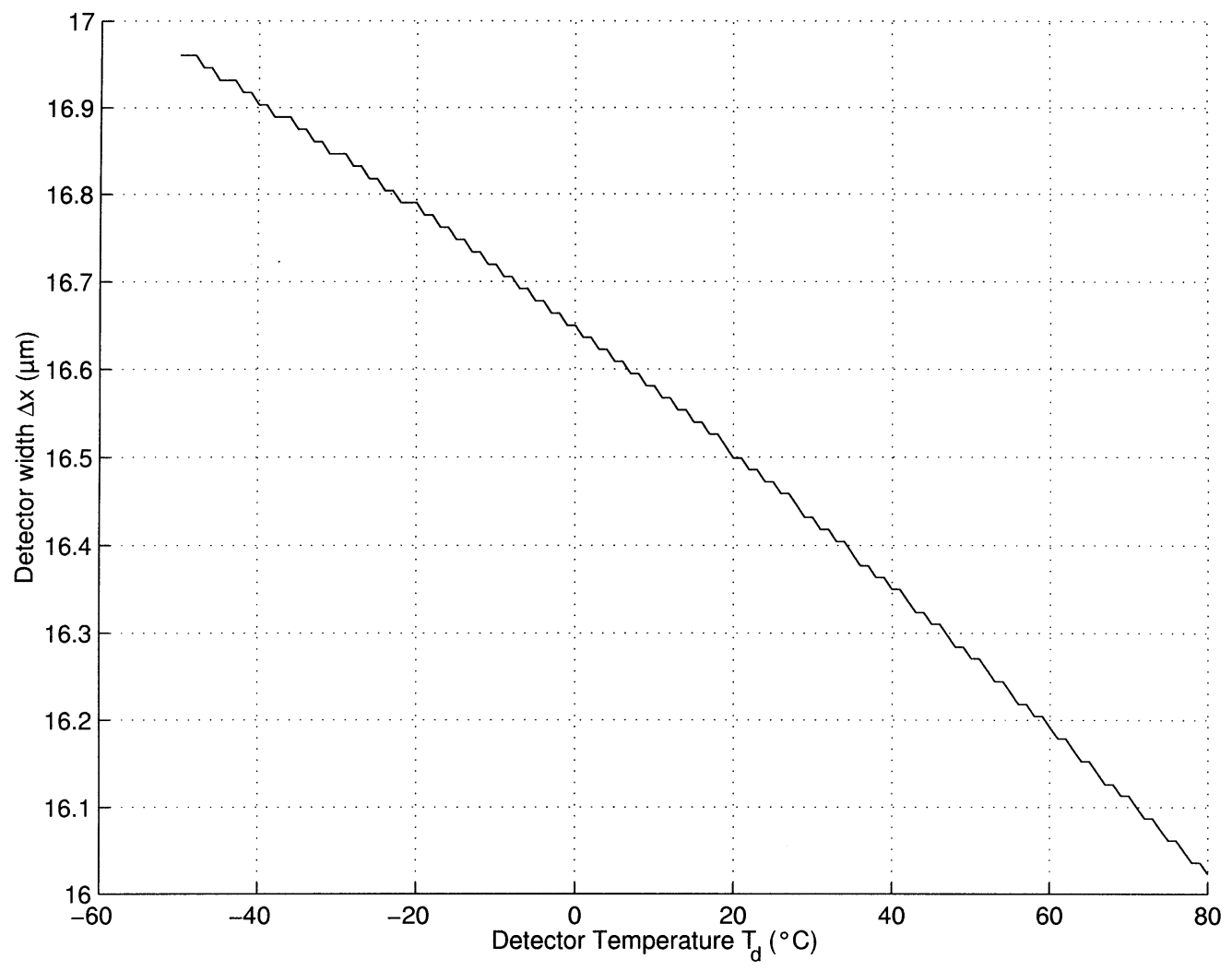

Fig. 18. $\Delta_{x}$ as a function of $T_{d}$.

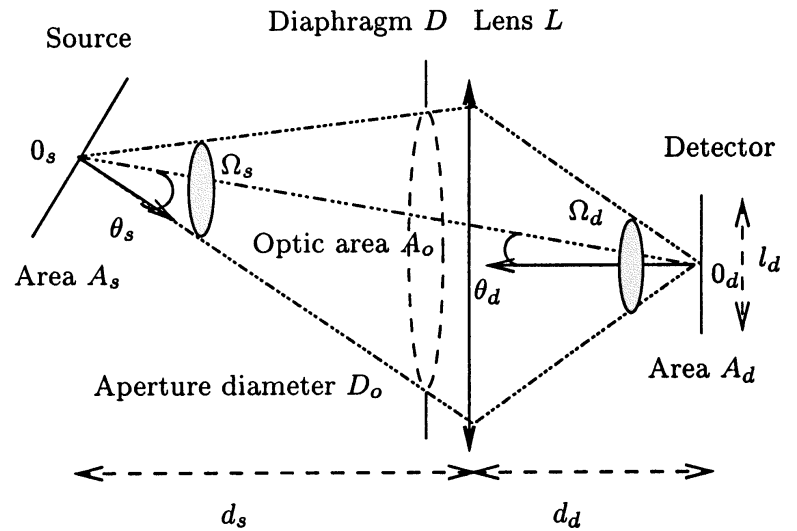

Fig. 19. Description of geometric situation.

In fact, the Fig. 19 shows the geometric situation between a hot source (black body) of area $A_{s}$ and sensor of area $A_{d}$ through the optical system of focal length $f$ and area $A_{o}$. The hot source sees optical area $A_{o}$ under the solid angle $\Omega_{s}=A_{o} / d_{s}^{2}$ (in the same way detector sees optical area $A_{o}$ under the solid angle $\left.\Omega_{d}=A_{o} / d_{d}^{2}\right) . \theta_{s}$ is the angle between the axis $0_{s}-0_{d}$ and the source normal $\vec{N}_{s}$ (respectively, $\theta_{d}$ with detector normal $\left.\vec{N}_{d}\right)$. In measurement situation, angles $\left(\theta s, \theta_{d}\right)$ are different from zero, the optical system is not focused and we have to correct the digital output signal measurement $I_{\text {Dmes }}$ by the geometric situation, as follows:

$$
I_{D}=\frac{I_{D m e s}}{\frac{A_{s} f^{2} \cos (\theta s)}{A_{d} d_{s}^{2}}}
$$

where $\theta_{d}, d_{s}$, and $A_{3}$ are provided by localization methods. After correction of $I_{\text {Dmes }}$ in relation (35), the radiometric model is inverted to measure the object temperature $T$ with respect to the corrected intensity $I_{D}$. Relation (36) provides real object temperature $T$

$$
T=\frac{B}{\ln \left(\frac{A_{3} \frac{G_{D} D^{t i}}{N^{2}} f\left(T_{d}\right)}{I_{D}-D\left(T_{d}\right)}+1\right)} .
$$

With any hot source, we must also modify $I_{D m e s}$ by emissivity value $\varepsilon$ at effective wavelength $\lambda_{e}$. It is better to work with short wavelength to decrease uncertainty on this emissivity. For example, in [22], a relative emissivity uncertainty $\Delta \varepsilon / \epsilon$ of $20 \%$ leads to a relative temperature uncertainty $\Delta T / T$ of $1.2 \%$ at wavelength $\lambda_{e}=1 \mu \mathrm{m}$ and of $12 \%$ at $\lambda_{e}=10 \mu \mathrm{m}$, respectively.

\section{CONCLUSION}

In this paper, we have described radiometric and geometric models taking into account detector temperature variations based on an uncooled low cost CCD camera operating in the NIR.

Using physical COD properties, we have analyzed the influence of wavelength and detector temperature fluctuations on the radiometric model [see (21)] usually used in cooled infrared thermographic systems. The temperature fluctuations (a) involve quantum efficiency variations due to light absorption and modifications of diffusion mechanisms [see (13)] and (b) 
affect sensor noise levels [see (18)] (especially dark current). Experimental calibration results confirm the radiometric model (21) with a COD camera based on a Sony detector over a detector temperature range of -30 to $50{ }^{\circ} \mathrm{C}$. Knowing the detector temperature with an external sensor, this model allows us to correct the object temperature by $10 \%$ (see Fig. 8) to compensate detector temperature fluctuations of $80{ }^{\circ} \mathrm{C}$. To obtain the same performances in function of detector temperature variation in terms of SNR or NETD, we propose to adjust the exposure time of the CCD detector. Figs. 9 and 10 show SNR and NETD, respectively, as a function of detector temperature $T_{d}$. An optimal point of both criteria at a detector temperature of $18{ }^{\circ} \mathrm{C}$ can be exhibited. Moreover, we can measure a hot object over the temperature range of 350 to $1000{ }^{\circ} \mathrm{C}$ with a precision lower than $\pm 25^{\circ} \mathrm{C}$ at $350{ }^{\circ} \mathrm{C}$.

On the other hand, by studying the MTF, we have analyzed the detector CCD spatial resolution properties in function of spectral band and detector temperature fluctuations. This analysis establishes that the spatial resolution decreases in NIR spectral band due to a diffusion term $\mathrm{MTF}_{\text {diff }}$ [see (28)]. This term $\left(\mathrm{MTF}_{\text {diff }}\right)$ depends on the absorption coefficient that is itself wavelength and detector temperature dependent. Therefore, we prove that the spatial cutoff frequency $f_{c}$ of a CCD detector decreases when the wavelength increases and the detector temperature decreases. As the cutoff frequency is inversely proportional to pixel size $\Delta x\left(f_{c}=1 / 2 \Delta_{x}\right)$, it is equivalent to work with an effective pixel size which is wavelength- and detector-temperature dependent. The properties are explained in geometric model (22). First, to consider wavelength effects, we have to calibrate the model in NIR spectral band to fit intrinsic parameters with effective pixel sizes. Then, using model (34), the detector temperature correction can be applied in geometric model (22) to compensate a relative error of $0.01 \%$ in a displacement of $100 \mathrm{~mm}$. This error could be higher with a thick substrate detector with greater diffusion effects.

We have characterized a low cost sensor to measure surface temperature field and dimensional characteristics. It could also be used in thermal treatment process of tools [23] or super plastic forming (SPF) of Titanium alloy sheets [24].

\section{APPENDIX}

According to [13], absorption photons coefficient is given as follows.

(a) For a photon energy $E$ between 1.2 and $2.2 \mathrm{eV}$

$\alpha=2750\left(\frac{E-1.1+510^{-7} T_{d}^{2}}{\exp \left(\frac{348}{T_{d}}\right)-1}+\frac{\left(E-1.16+510^{-7} T_{d}^{2}\right)^{2}}{1-\exp \left(\frac{-348}{T_{d}}\right)}\right)$.

(b) For a photon energy $E$ between 2.2 and $2.5 \mathrm{eV}$

$$
\alpha=\alpha\left(2.2 e V ; T_{d}\right)\left(\frac{7447.3}{\alpha\left(2.2 e V ; T_{d}\right)}+0.4491\right)^{\left(\frac{E}{0.3-7.3333}\right)}
$$

\section{ACKNOWLEDGMENT}

The authors would like to thank J. J. Orteu for his helpful comments, as well as Referee.

\section{REFERENCES}

[1] Y. Le Maoult, S. Baleix, P. Lours, and G. Bernhart, "Application industrielle de la thermographie infrarouge au comportement des outillages de formage superplastique," in Congrès National sur les applications de la thermographie dans les industries mécaniques. Senlis, France, 1999.

[2] D. Garcia and J. J. Orteu, "3D deformation measurement using stereo-correlation applied to the forming of metal or elastomer sheets," in Int. Workshop Video-Controlled Mater. Testing In-Situ Microstructural Characterization. Nancy, France, Nov. 1999.

[3] S. Claudinon, P. Lamesle, J. J. Orteu, and R. Fortunier, "Continuous in-situ measurement of quenching distortions using computer vision," J. Mater. Processing Technol., vol. 122, pp. 69-81, Mar., 2002.

[4] G. J. Zissis, Ed., The Infrared \& Electro-Optical Systems Handbook. Bellingham, WA: SPIE, 1992, vol. 1.

[5] F. Moreau, "Thermographie Proche Infrarouge par Caméras CCD et Application aux Composants de Premiére Paroi du Tokamak TORE SUPRA,” Ph.D. dissertation, Univ. Saint Jérôme, Marseille, France, June 1996.

[6] F. Mériaudeau, E. Renier, and F. Truchetet, "Uncertainty committed on temperature measurement," in Congr. Int. Métrologie, vol. 1, Nimes, France, Oct. 1995, pp. 189-194.

[7] E. Healey and K. Ragava, "Radiometric CCD camera calibration and noise estimation," IEEE Trans. Pattern Anal. Machine Intell., vol. 16, p. 267, Mar. 1994.

[8] R. Y. Tsai, "A versatile camera calibration technique for hight-accuracy 3D machine vision metrology using off-the-shelf tv cameras and lenses," IEEE J. Robot. Automat., vol. RA-3, Aug. 1987.

[9] P. Saunders and T. Ricolfi, "The characterization of a CCD camera for the purpose of temperature measurement," in Int. Symp. Temperature Thermal Meas. Ind. Sci. (TEMPMEKO), Torino, Italy, Sept. 1996, pp. 329-334.

[10] M. Orgeret, Les Piles Solaires, le Composant et ses Applications. Paris, France: Editions Masson, 1985.

[11] F. H. Gaensslen, V. L. Rideout, E. J. Walker, and J. J. Walker, "Very small MOSFET's for low temperature operation," IEEE Trans. Electron Devices, vol. ED-24, pp. 218-229, Mar. 1977.

[12] 75222 “Tech. Rep.," Texas Instruments Incorpored, Central Research Laboratories, Dallas, TX, May 1975.

[13] G. Roiland, "Etude des variations de rendement quantique interne d'un détecteur CCD en fonction de la température," Rev. Phys. Appliquée, no. 20, pp. 651-659, Sept. 1985.

[14] J. M. Lavest, M. Viala, and M. Dhome, "Do you really need an accurate calibration pattern to achieve a reliable camera calibration," in Eur. Conf. Comput. Vision (ECCV), vol. 1, Freiburg, Germany, June 1998, pp. $158-174$.

[15] G. Weng, S. Ma, and M. Herniou, "Camera calibration with distorsion models and accuracy evaluation," IEEE Trans. Pattern Anal. Machine Intell., vol. 14, no. 10, pp. 965-980, Oct. 1992.

[16] M. Devy, V. Garric, and J. J. Orteu, "Camera calibration from multiple views of a 2D object, using a global non linear minimization method," in IEEE/RSJ Int. Conf. Intell. Robots Syst. (IROS), Grenoble, France, Sept. 8-12, 1997.

[17] A. Fusiello, "Uncalibrated euclidean reconstruction a review," Image Vision Comput., vol. 18, no. 6-7, pp. 555-563, May 2000.

[18] M. A. Abidi and T. Chandra, "A new efficient and direct solution for pose estimation using quadrangular targets algorithm and evaluation," IEEE Trans. Pattern Anal. Machine Intell., vol. 17, p. 534, May 1995.

[19] M. C. Dudzik, Ed., The Infrared 81 Electro-Optical Systems Handbook. Bellingham, WA: SPIE, 1992, vol. 4.

[20] G. C.Gerald C. Hoist, Ed., CCD Arrays Cameras and Displays. Bellingham, WA: SPIE, 1998.

[21] D. H. Sieb, "Carrier diffusion degradation of modulation transfer function in charge coupled imagers," IEEE Trans. Electron Devices, vol. ED-21, pp. 210-217, may 1974.

[22] J. Martinet, La Mesure des Températures par Rayonnement Thermique. Paris, France: Bureau National de Métrologie, 1981.

[23] S. Claudinon, "Contribution à L'étude de la Distorsion des Aciers au Traitement Thermique : Suivi en Continu par Vision Artificielle et Simulation Numérique," Ph.D. dissertation, Ecole des Mines Paris, Février, France, 1999. 
[24] S. Baleix, "Oxydation et Écaillage D’alliages Réfractaires Moules Pour Outils de Formage Superplastiqne,” Ph.D. dissertation, Université Paul Sabatier, Toulouse, France, Dec. 1999.

Thierry Sentenac received the M.S. degree in computer science from INP, Toulouse, France, in 1992. He is currently pursuing the Ph.D. degree at the Laboratoire d'Analyze et d'Architecture des Systèmes (LAAS)-CNRS, Toulouse.

From 1992 to 1995, he was a Research Engineer at LAAS-CNRS. Since 1995, he has been an Associate Professor and he is Head of the Electrical Engineering Department, Ecole des Mines d'AIbi, Albi, France, specializing in process engineering. His current research interests cover CCD image processing, computer vision, and optoelectronics.

Yannick Le Maoult received the M.S. degree in physical measurements from INP, Grenoble, France, in 1987, and the Ph.D. degree from the University de Provence, Marseille, France, in 1992.

Since 1992, he has been an Associate Professor at the Ecole des Mines d'Albi, Albi, France, and a French Grande Ecole specializing in process engineering. $\mathrm{He}$ is a member of the Computer Vision and IR Thermography Group (Material Research Center) and his fields of interest include radiative transfer, optical measurements, metrology, and inverse methods. Particularly, he is involved in infrared measurements, such as thermography or pyrometry, applied to material processing or reactive flows.
Guy Rolland has been an Engineer with the French National Center for Space Research (CNES), France, since 1978. From 1978 to 1987, he worked in the field of characterization and physical modeling of CCDs and other detectors for earth observation and scientific satellites. From 1987 to 1999, he concentrated on the field of modeling CCD and active electronics components by means of finite elements methods (process and devices simulations). Since 1999, he has been working in the field of radiation effects on components and systems. One of his research themes today is the effect of space radiation on CCDs. He also teaches physics of semiconductors imaging and components in Toulouse, France, university and engineer schools.

Michel Devy received the M.S. degree in computer science engineering from IMAG, Grenoble, France, in 1976, and the Ph.D. degree from the Laboratoire d'Analyze et d'Architecture des Systèmes (LAAS)-CNRS, Toulouse, France, in 1980.

Since 1980, he has participated in the Robotics and Artificial Intelligence Group of LAAS-CNRS. His research is devoted to the application of computer vision in automation and robotics. He has been involved in numerous national and international projects regarding manufacturing applications, mobile robots for space exploration or for civil safety, and 3-D vision for intelligent vehicles or medical applications. He is now Research Director at CNRS, Head of the Perception Area in the Robotics Group, and his main scientific topics concern perception for mobile robots in natural or indoor environments. 